\title{
Distribución de las mariposas diurnas (Lepidoptera: Hesperioidea y Papilionoidea) del Estado de México, México
}

\author{
Claudia Hernández-Mejía, Isabel Vargas-Fernández, Armando Luis-Martínez \& Jorge Llorente- \\ Bousquets* \\ Museo de Zoología, Facultad de Ciencias, Universidad Nacional Autónoma de México. Apartado Postal 70-399, \\ México 04510, D.F. MÉXICO; claudia_hernandezm@hotmail.com, ivf@hp.fciencias.unam.mx, alm@hp.fciencias. \\ unam.mx, jlb@hp.fciencias.unam.mx (correspondencia)*Autor responsable.
}

Recibido 02-VIII-2007. Corregido 30-VI-2008. Aceptado 31-VII-2008.

\begin{abstract}
Distribution of butterflies (Lepidoptera: Hesperioidea and Papilionoidea) from Mexico State, Mexico. The State of Mexico is a region with great biological diversity, owing to its geographical and ecological features. Regarding Hesperioidea and Papilionoidea, $15 \%$ of the Mexican species are recorded in the State of Mexico, $17 \%$ of which are endemic to the country. A checklist of the two superfamilies for the State of Mexico was integrated, based on published literature and databases at the Museo de Zoología of the Facultad de Ciencias, UNAM. The checklist is composed by six families, 22 subfamilies, 197 genera and 325 species (95 Hesperiidae, 19 Papilionidae, 35 Pieridae, 54 Lycaenidae, 20 Riodinidae, and 102 Nymphalidae). A list of each species is presented, including collecting localities, flight month, and whether data correspond to scientific collection records or literature. Rev. Biol. Trop. 56 (3): 1309-1341. Epub 2008 September 30.
\end{abstract}

Key words: collections, Nearctic, Neotropical, municipality, species list.

Del Estado de México son escasos y puntuales los trabajos lepidopterológicos que se han realizado, pese a su alta riqueza biológica resultado de su accidentada topografía, diversidad climática y vegetacional, y ser área crítica de la zona de transición entre las regiones Neártica y Neotropical. Estos factores hacen posible la coexistencia de especies de ambas regiones, incrementando su biodiversidad (Luna et al. 2007).

La primera publicación sobre la recolecta de mariposas en el Estado de México es la Biologia Centrali Americana de Godman y Salvin (1878-1901). En ella se cita el municipio de Amecameca como localidad de recolecta de 11 especies (una papiliónida, tres piéridas, dos licénidas y cinco ninfálidas). Trabajos posteriores en los que se incluyen ejemplares recolectados en el estado son los encabezados por Vázquez (1951), Gibson y Carrillo (1959), Higgins (1960), Miller (1974), Domínguez y Carrillo (1976), De la Maza (1976, 1980), Beutelspacher (1976, 1983), De la Maza y De la Maza (1978), De la Maza y Turrent (1978, 1985), Descimon y Mast (1979), Hernández et al. (1981), Jenkins (1983, 1986), Miller y De la Maza (1984), Kendall y McGuire (1984), Calvert y Brower (1986) y Steinhauser (1989). Sin embargo, autores que han contribuido considerablemente son: Guzmán (1976), quien citó 45 especies para Chalma; Barrera y DíazBatres (1977), quienes citaron para Tejupilco 29 especies; Barrera y Romero (1986) registraron 35 especies para San Rafael, y De la Maza (1987) que en su libro "Mariposas de México" incluyó 39 especies de mariposas diurnas procedentes de Atizapán de Zaragoza, Donato Guerra, Santo Tomás, Tejupilco, Toluca y Valle de Bravo.

Como puede advertirse de tales antecedentes, las investigaciones se enfocaban sobre estudios faunísticos locales, la búsqueda de 
especies raras o de gran valor, así como muestras para estudios taxonómicos. De esta forma, las recolectas eran ocasionales o durante periodos muy breves. En la actualidad, los métodos sobre el estudio de mariposas se han perfeccionado, considerando además aspectos biológicos, ecológicos, biogeográficos y conductuales, entre otros. Esas recolectas se deben de efectuar de manera sistemática, lo que genera trabajos faunísticos formales y comparables. Recientemente, Luna-Reyes y Llorente-Bousquets (2004) realizaron un estudio sobre la distribución local, altitudinal y por comunidad vegetacional, de las mariposas diurnas en 37 localidades del área que comprende la Sierra Nevada (Parque Nacional Izta-Popo), ubicada entre los 1900 y los $3800 \mathrm{msnm}$. Ellos integraron una lista con 75 especies, de las cuales 34 fueron nuevos registros para el área y cuatro para el estado. Un trabajo similar es el de Hernández-Mejía (2005) en el municipio de Malinalco (1 $800 \mathrm{msnm}$ ), área donde predomina el bosque tropical caducifolio, cuya lista generada en un trabajo faunístico formal se integró por 213 especies de hesperioideas y papilionoideas, de las cuales 157 fueron nuevos registros (63 regionales y 94 estatales).

\section{MATERIALES Y MÉTODOS}

Área de estudio: El Estado de México, con una extensión territorial de $21355 \mathrm{~km}^{2}$ distribuida en 122 municipios, ocupa el 1.1\% del territorio Nacional. Se ubica entre los paralelos $20^{\circ} 17^{\prime}-18^{\circ} 22^{\prime} \mathrm{N}$ y $98^{\circ} 36^{\prime}-100^{\circ} 37^{\prime} \mathrm{W}$. Colinda al norte con Michoacán de Ocampo, Querétaro de Arteaga e Hidalgo; al este con Hidalgo, Tlaxcala, Puebla, Morelos y el Distrito Federal; al sur con Morelos y Guerrero; al oeste con Guerrero y Michoacán de Ocampo (INEGI 2004). Desde el punto de vista geológico, está compuesto de modo mayoritario por dos provincias geológicas, en el norte básicamente por el Eje Neovolcánico y porciones del sur de la Mesa Central (Llanuras y Sierras de Querétaro e Hidalgo, Mil Cumbres y Lagos y Volcanes del Anáhuac), y en el sur esencialmente por la
Sierra Madre del Sur y la Depresión del Balsas (sierras y valles guerrerenses). Las elevaciones de mayor altitud son los volcanes Popocatépetl (5 $500 \mathrm{~m}$ ), Iztaccíhuatl (5 $220 \mathrm{~m}$ ) y Xinantécatl (4 $680 \mathrm{~m}$ ); la localidad de menor altitud (400 m) se ubica en el municipio de Tlatlaya (Vilchis 1992).

La heterogeneidad en los rasgos topográficos, climáticos y edáficos ha permitido que se establezcan diversas comunidades vegetales, las principales conforme a la clasificación de Rzedowski (1978) son: bosque de coníferas, bosque espinoso, bosque de galería, bosque mesófilo de montaña, bosque de Quercus, bosque tropical caducifolio, matorral xerófilo, pastizal y vegetación acuática; desafortunadamente, la mayoría de ellas exhibe algún grado de alteración ecológica, a veces muy profunda, a causa de las actividades humanas.

Base de datos: Se consultó la base de datos 'MARIPOSA' del Museo de Zoología “Alfonso L. Herrera" de la Facultad de Ciencias UNAM, creada en 1987 (Luis et al. 2005) y se obtuvieron los datos del Estado de México, a lo cual se adicionó la información producida de los trabajos de Luna-Reyes y Llorente-Bousquets (2004) y Hernández-Mejía (2005), obteniendo un total de 1507 registros procedentes de 30 municipios del Estado de México. Para un examen detallado de la conformación y estructura de la base de datos 'MARIPOSA', véanse los trabajos de Luis et al. (2003, 2005).

Las publicaciones nacionales e internacionales en que se menciona la entidad como localidad de recolecta de mariposas son: Acta Zoológica Mexicana, Anales del Instituto de Biología UNAM, Biologia Centrali Americana, Boletín y Revista de la Sociedad Mexicana de Lepidopterología, Bulletin of the Allyn Museum, Fitófilo, Folleto Misceláneo del Instituto Nacional de Investigaciones Agrícolas, Journal of the Lepidopterists' Society, y el libro "Mariposas Mexicanas", entre las principales. Todas éstas se examinaron para la elaboración de la base de datos 'MARIPOSA' (Luis et al. 2003, 2005), que se utilizó en este trabajo. 


\section{RESULTADOS}

Al analizar la información de la base de datos 'MARIPOSA', al menos 1210 de los ejemplares registrados se albergan en alguna de las siguientes colecciones (Cuadro 1): AME, AMNH, CAS, CDM, CMNH, CNIN, COEE, CTE, DGSV, FES-Z, HOLLA, INIA, LACM, MCLB, MZFC, UCB y USNM. La colección que posee el mayor número de registros de Hesperioidea y Papilionoidea (654) y el mayor número de taxones (247), entre especies y subespecies, es la Colección Nacional de Insectos del Instituto de Biología, UNAM. En el Cuadro 1 se sintetiza la información recabada en las principales colecciones nacionales e internacionales en que se haya depositado material recolectado en el Estado de México.

Posteriormente, se obtuvieron 1507 registros de hesperioideas y papilionoideas procedentes del Estado de México, las cuales han sido recolectadas en los municipios de Almoloya de Alquisiras, Amecameca, Atizapán, Atizapán de Zaragoza, Atlauta, Chiautla, Cuautitlán, Donato Guerra, Ixtapan de la Sal, Jilotepec, Malinalco, Naucalpan, Nicolás Romero, Ocuilan de Arteaga, El Oro, Santo Tomás, Tejupilco, Temascaltepec, Tenancingo, Teotihuacán, Texcoco, Tlalnepantla de Baz, Toluca, Tonatico, Tultepec, Tultitlán, Valle de Bravo, Villa Guerrero, Zacualpan y Zumpango (Cuadro 2), de los otros 92 municipios (casi el

\section{CUADRO 1}

Acrónimos de las colecciones, nombres de las instituciones o colecciones, número de registros y número de especies y subespecies de Hesperioidea y Papilionoidea del Estado de México en colecciones nacionales y extranjeras

TABLE 1

Acronyms for collections, name of the institution or collection, number of records, and number of species and subspecies of Hesperioidea and Papilinoidea from the State of Mexico in national and foreign collections

Registros

$\begin{array}{cl}\text { AME } & \text { Allyn Museum of Entomology, Sarasota, FL (hasta 1985) } \\ \text { AMNH } & \text { American Museum of Natural History, New York, NY } \\ \text { CAS } & \text { California Academy of Sciences, San Francisco, CA } \\ \text { CDM } & \text { Colección Particular Familia De la Maza } \\ \text { CMNH } & \text { Carnegie Museum of Natural History, Pittsburgh, PA } \\ \text { CNIN } & \text { Colección Nacional de Insectos, Instituto de Biología, UNAM } \\ \text { COEE } & \text { Colección Entomológica de la Oficina de Estudios Especiales, S.A.G. México, D.F. } \\ \text { CTE } & \text { Colección Escalante } \\ \text { DGSV } & \text { Dirección Nacional de Sanidad Vegetal, México } \\ \text { FES-Z } & \text { Museo de Zoología, Facultad de Estudios Superiores, Zaragoza, UNAM } \\ \text { HOLLA } & \text { Colección Particular Richard Holland } \\ \text { INIA } & \text { Instituto Nacional de Investigaciones Agrícolas } \\ \text { LACM } & \text { Los Angeles County Museum of Natural History, CA } \\ \text { MCLB } & \text { McGuire Center for Lepidoptera and Biodiversity, Gainesville, FL } \\ \text { MZFC } & \text { Museo de Zoología “Alfonso L. Herrera” de la Facultad de Ciencias, UNAM } \\ \text { UCB } & \text { University of California, Berkeley, CA } \\ \text { USNM } & \text { National Museum of Natural History of Washington, DC } \\ \end{array}$

\begin{tabular}{cc}
86 & 47 \\
10 & 10 \\
3 & 3 \\
1 & 1 \\
3 & 3 \\
654 & 247 \\
11 & 11 \\
1 & 1 \\
3 & 3 \\
75 & 75 \\
1 & 1 \\
7 & 7 \\
1 & 1 \\
51 & 28 \\
279 & 116 \\
4 & 4 \\
20 & 17 \\
1210 & 308 \\
\hline
\end{tabular}

especies /
subespecies 
CUADRO 2

Municipios del Estado de México que presentan registros de Hesperioidea y Papilionoidea

TABLE 2

Municipalities in the state of Mexico from which we have records of Hesperioidea and Papilionoidea

\begin{tabular}{|c|c|c|c|}
\hline Claves & Municipios & Registros & Especies/subespecies \\
\hline ALQ & Almoloya de Alquisiras & 12 & 11 \\
\hline AME & Amecameca & 18 & 14 \\
\hline ATZ & Atizapán & 15 & 13 \\
\hline $\mathrm{AZ}$ & Atizapán de Zaragoza & 46 & 28 \\
\hline ATL & Atlauta & 3 & 3 \\
\hline CHIA & Chiautla & 21 & 19 \\
\hline CUA & Cuautitlán & 5 & 5 \\
\hline DG & Donato Guerra & 1 & 1 \\
\hline EM & Municipio no definido & 354 & 132 \\
\hline IXS & Ixtapan de la Sal & 8 & 8 \\
\hline JIL & Jilotepec & 4 & 4 \\
\hline MALI & Malinalco & 648 & 240 \\
\hline NAU & Naucalpan & 5 & 5 \\
\hline NR & Nicolás Romero & 4 & 3 \\
\hline $\mathrm{OA}$ & Ocuilan Arteaga & 69 & 49 \\
\hline ORO & El Oro & 1 & 1 \\
\hline ST & Santo Tomás & 19 & 17 \\
\hline TEJ & Tejupilco & 36 & 32 \\
\hline TEM & Temascaltepec & 64 & 36 \\
\hline TEN & Tenancingo & 9 & 8 \\
\hline TEO & Teotihuacán & 5 & 4 \\
\hline TEX & Texcoco & 27 & 19 \\
\hline TLAL & Tlalnepantla & 1 & 1 \\
\hline TOL & Toluca & 29 & 17 \\
\hline TON & Tonatico & 8 & 7 \\
\hline TUL & Tultepec & 3 & 3 \\
\hline TULT & Tultitlán & 1 & 1 \\
\hline $\mathrm{VB}$ & Valle de Bravo & 24 & 22 \\
\hline VG & Villa Guerrero & 22 & 17 \\
\hline ZAC & Zacualpan & 42 & 28 \\
\hline \multirow[t]{2}{*}{ ZUM } & Zumpango & 3 & 3 \\
\hline & & 1507 & 325 \\
\hline
\end{tabular}




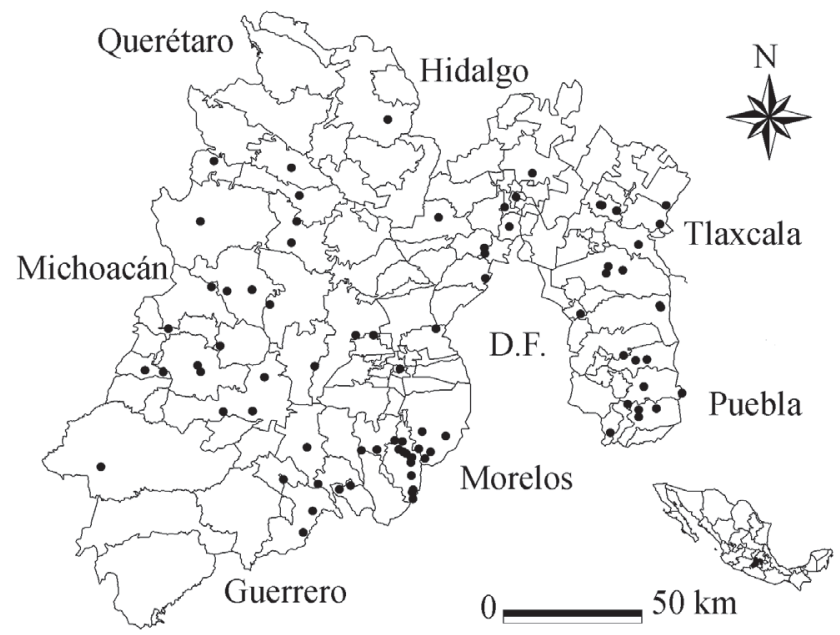

Fig. 1. Mapa de las localidades de recoleca de Hesperioidea y Papilionoidea en el Estado de México.

Fig. 1. Map of the localities of collects of Hesperioidea and Papilionoidea in the State of Mexico.

75\%) que componen el estado sólo se tienen datos dispersos de la lepidopterofauna que los constituye. En la Figura 1 se muestran las localidades de recolecta que han sido georreferenciadas. De este mapa, se deduce que es necesario realizar estudios lepidopterofaunísticos en municipios con diversas condiciones ecológico-geográficas y que no presentan registros de Hesperioidea y Papilionoidea, principalmente en el norte y suroeste, donde aún existen zonas conservadas.

Con base en la información recabada se registraron 325 especies, cifra que representa el 15\% de las que ocurren en México (2 103); el $17 \%$ de tales taxones son endémicos para el país. El municipio que posee el mayor número de especies es Malinalco (240), sitio en el que se realizaron recolectas sistemáticas en el transcurso de un año en doce localidades ubicadas entre los 1000 y 2000 msnm (Cuadro 2) (Hernández-Mejía et al. 2008).

La familia con mayor riqueza es Nymphalidae (102 especies), seguida de Hesperiidae (95), Lycaenidae (54), Pieridae (35), Riodinidae (20) y Papilionidae (19). En el Cuadro 3 se sintetiza la riqueza de taxones por familia. A partir de toda la información reunida se elaboró el Apéndice 1.

Para el arreglo taxonómico se siguió la nomenclatura adoptada por Warren (2000) y Llorente-Bousquets et al. (2006), en sus listas sobre Hesperioidea y Papilionoidea, respectivamente.

\section{DISCUSIÓN}

De acuerdo con la Figura 1, se observa que debido a su posición geográfica y los estados que lo limitan, el Estado de México debería presentar una mayor diversidad; sin embargo, la carencia de trabajos faunísticos formales, nos permite afirmar que falta mucho trabajo de campo por realizar, ya que algunas regiones del norte y suroeste se desconocen por completo. Es importante que al realizar los inventarios se considere a Hesperiidae, familia poco estudiada en la entidad, la cual constituye cerca del $40 \%$ de las especies de mariposas presentes en la República Mexicana, y en general en cualquier estudio regional (G. Lamas y O. Mielke, com. pers.). 
CUADRO 3

Distribución numérica por niveles taxonómicos para Hesperioidea y Papilionoidea en el Estado de México

TABLE 3

Number distribution by taxonomic levels for Hesperioidea and Papilionoidea in the State of Mexico

$\begin{array}{lccc}\text { Familias } & \text { Subfamilias } & \text { Géneros } & \text { Especies/subespecies } \\ \text { Hesperiidae } & 4 & 64 & 95 \\ \text { Papilionidae } & 2 & 8 & 19 \\ \text { Pieridae } & 3 & 23 & 35 \\ \text { Lycaenidae } & 2 & 36 & 54 \\ \text { Riodinidae } & 2 & 12 & 20 \\ \text { Nymphalidae } & 9 & 54 & 102 \\ \text { Total } & 22 & 197 & 325\end{array}$

CUADRO 4

Riqueza estatal de Papilionoidea

TABLE 4

State richness of Papilionoidea

\begin{tabular}{|c|c|c|c|c|c|c|c|c|c|c|c|c|c|c|c|}
\hline Taxón/Estado & $\mathrm{DN}$ & $\mathrm{IN}$ & JAY & JAL & $\mathrm{OL}$ & $\mathrm{MICH}$ & GRO & MOR & OAX & CHIS & HGO & PUE & TLAX & QRO & 1015 \\
\hline pilionidae & 16 & 21 & 25 & 26 & 30 & 29 & 32 & 26 & 51 & 42 & 27 & 34 & 4 & 15 & 19 \\
\hline erid & 45 & 36 & 31 & 44 & 37 & 44 & 40 & נו & 04 & $0 /$ & 40 & 47 & 10 & 54 & 35 \\
\hline ycaenidae & 68 & 60 & 50 & 90 & 7 & 122 & 132 & 80 & 64 & 180 & 4 & 118 & 7 & 5 & 54 \\
\hline iodinidae & 34 & 33 & 34 & 51 & 8 & 53 & 67 & 42 & 125 & 147 & 33 & 65 & 1 & 9 & 20 \\
\hline Nymphalidae & 105 & 107 & 145 & 178 & 140 & 173 & 224 & 148 & 388 & 396 & 169 & 228 & 20 & 48 & 102 \\
\hline apilionoidea & 268 & 257 & 300 & 389 & 322 & 421 & 501 & 342 & 792 & 832 & 349 & 494 & 35 & 131 & 230 \\
\hline México & $22 \%$ & $21 \%$ & $25 \%$ & $32 \%$ & $27 \%$ & $35 \%$ & $42 \%$ & $28 \%$ & $66 \%$ & $70 \%$ & $29 \%$ & $41 \%$ & $4 \%$ & $11 \%$ & $19 \%$ \\
\hline
\end{tabular}

Datos tomados de Llorente-Bousquets et al. (2006), para Hesperioidea no hay datos disponibles, solo registros ocasionales.

*Para el Estado de México se añadieron los registros de Luna-Reyes \& Llorente-Bousquets (2004) y de Hernández-Mejía (2005).

Adicionalmente, en el Cuadro 4 se presenta la riqueza de especies de Papilionoidea por Estado, tanto en la vertiente del Pacífico mexicano, como en los estados que hacen frontera con éste. En este cuadro se aprecia que el Estado de México ocupa el lugar 13, solo mayor que el Distrito Federal, Querétaro y Tlaxcala; pero en estos dos últimos es donde se ha realizado menor esfuerzo de recolecta y de donde no hay estudios faunísticos formales, teniendo solo muestreos ocasionales. El Estado de México posee el 19\% de la riqueza de especies de Papilionoidea de México (1 190); respecto a Chiapas y Oaxaca, los estados de mayor diversidad, las Papilionoidea muestran el 27 y 29\% respectivamente. En el Cuadro 5, se muestra el número de localidades, registros e individuos, que se tienen incluidos en la base 
CUADRO 5

Número de localidades, registros e individuos que se tienen en la base de datos 'MARIPOSA', para el Estado de México y los Estados con que hace frontera

TABLE 5

Number of localities, registries and individuals that have in the data base 'MARIPOSA', for the State of Mexico and the States whereupon it makes border

\begin{tabular}{|c|c|c|c|c|}
\hline Estados & Localidades & Registros* & Individuos & Especies \\
\hline Michoacán & 319 & 176902 & 181878 & 421 \\
\hline Guerrero & 274 & 24044 & 25174 & 501 \\
\hline Hidalgo & 156 & 4219 & 4503 & 349 \\
\hline Puebla & 145 & 13340 & 13590 & 494 \\
\hline Morelos & 123 & 11684 & 12219 & 342 \\
\hline Estado de México & 100 & 1290 & 1528 & 230 \\
\hline Querétaro & 21 & 450 & 464 & 151 \\
\hline Tlaxcala & 10 & 20 & 20 & 53 \\
\hline Distrito Federal & 82 & 6703 & 6909 & 116 \\
\hline
\end{tabular}

*Datos tomados de Luis et al. (2005) y Llorente-Bousquets et al. (2006). *Un registro contiene uno o más individuos.

de datos 'MARIPOSA', se advierte el desconocimiento general que se tiene de los cuatro últimos estados, entre los que se encuentra el Estado de México; para éstos es necesario realizar un mayor esfuerzo de trabajo de campo con base en recolecciones sistemáticas, en estudios faunísticos formales en áreas de gran heterogeneidad geográfica y biótica, como ya lo han hecho para Guerrero (Vargas et al. 1994) y Jalisco-Colima (Vargas et al. 1999).

\section{AGRADECIMIENTOS}

Al Instituto de Biología UNAM, especialmente a Adolfo Ibarra, Cristina Mayorga y Harry Brailovsky, por permitirnos el acceso a la Colección Nacional de Insectos, sección Lepidoptera, y el apoyo para la realización de parte de este trabajo. A Andrew Warren por la revisión y correcciones efectuadas a la lista de Hesperioidea. A todos los curadores que nos permitieron el acceso a las colecciones con las cuales se elaboró la base de datos 'MARIPOSA' y a los proyectos PAPIIT IN 218502, $212006 \mathrm{y}$ 203509.

\section{RESUMEN}

El Estado de México posee características geográfico-ecológicas que lo hacen una región de gran diversidad biológica; respecto a Hesperioidea y Papilionoidea posee el $15 \%$ de las especies registradas para México, del cual el $17 \%$ son endémicas para el país. Con base en la información bibliográfica y la consulta de la base de datos del Museo de Zoología de la Facultad de Ciencias, UNAM, se integró la lista de las dos superfamilias para el Estado de México; esta se compone de seis familias, 22 subfamilias, 197 géneros y 325 especies (95 Hesperiidae, 19 Papilionidae, 35 Pieridae, 54 Lycaenidae, 20 Riodinidae y 102 Nymphalidae). De cada especie se anexó la lista de localidades de recolecta, vuelo, la colección donde están depositados los ejemplares, o la cita de la cual se tomó el dato.

Palabras clave: colecciones, lista de especies, municipios, Neártica, Neotropical.

\section{REFERENCIAS}

$\S$ Adams, J.K. 1983. An old first United States record finally published: Papilio victorinus (Papilionidae) in Laredo, Texas. Jour. Lep. Soc. 37: 318-318.

Barrera, A. \& E. Díaz-Batres. 1977. Distribución de algunos lepidópteros de la Sierra de Nanchititla, México, con especial referencia a Thisiphone maculata Hpff. 
(Insecta: Lepidoptera). Rev. Soc. Mex. Lep. 3: $17-28$.

Barrera, T.G. \& L.H. Romero. 1986. Estudio faunístico de lepidópteros (Superfamilia Papilionoidea) en un bosque mesófilo de montaña en Cascada de Los Diamantes, San Rafael, Estado de México. Tesis de Biología. Escuela Nacional de Estudios Profesionales Zaragoza, Universidad Nacional Autónoma de México, México D.F, México. 58 p.

Beutelspacher, C.R. 1976. Nuevas formas de papiliónidos mexicanos. Rev. Soc. Mex. Lep. 2: 61-70.

Beutelspacher, C.R. 1983. La familia Pieridae (Lepidoptera) en el Estado de Nuevo León, México. An. Inst. Biol. UNAM (Zoología) 53: 367-378.

$\S$ Beutelspacher, C.R. \& W.H. Howe. 1984. Mariposas de México. I. Introducción y generalidades. Superfamilia Papilionoidea. La Prensa Médica Mexicana. México, D.F, México. 128 p.

Calvert, W.H. \& L.P. Brower. 1986. The location of Monarch butterfly (Danaus plexippus L.) overwintering colonies in Mexico in relation to topography and climate. Jour. Lep. Soc. 40: 164-187.

De la Maza, R.R. 1976. Notas sobre la variabilidad de Anteros carausius Westw. (Riodinidae). Rev. Soc. Mex. Lep. 2: 71-72.

De la Maza, R.R. \& R.E. De la Maza. 1978. Notas sobre la familia Papilionidae de México I.- San Nicolás Tolentino, Mex. (Lep.). Bol. Inf. Soc. Mex. Lep. 4: 3-7.

De La Maza, R.E. \& R. Turrent D. 1978. Notas sobre la familia Papilionidae en México (Lep.) III.- Área del Valle de México. Bol. Inf. Soc. Mex. Lep. 4: 5-14.

De la Maza, R.E. 1980. Las poblaciones centroamericanas de Parides erithalion (Boisd.) (Papilionidae: Troidini). Rev. Soc. Mex. Lep. 5: 51-74.

De la Maza, R.E. \& R. Turrent D. 1985. Mexican Lepidoptera, Eurytelinae I. Publ. Espec. Rev. Soc. Mex. Lep. 4: 1-44.

De la Maza, R.R. 1987. Mariposas Mexicanas. Fondo de Cultura Económica, México D.F, México. 301 p.

Descimon, H. \& J. Mast. 1979. Contribución al conocimiento de las Nymphalidae Neotropicales: Epiphile adrasta Hewitson. Rev. Soc. Mex. Lep. 5: 39-47.

Domínguez Y. \& J.L. Carrillo. 1976. Lista de Insectos en la Colección Entomológica del Instituto Nacional de Investigaciones Agrícolas, S.A.G. México. Foll. Misc. Secr. Agr. Ganad. 29: 142-149.
Gibson, W.W. \& J.L. Carrillo. 1959. Lista de insectos en la Colección Entomológica de la Oficina de Estudios Especiales, S.A.G. México. Foll. Misc. Secr. Agric. Ganad. 9: 1-254.

Godman, F.D. \& O. Salvin. 1878-1901. Biologia Centrali Americana. Zoología, Insecta, Lepidoptera Rhopalocera. London, Taylor \& Francis. Vols. I-III.

Guzmán, P.E. 1976. Algunas observaciones sobre lepidópteros de Chalma, Estado de México. Rev. Soc. Mex. Lep. 2: 49-51.

Higgins, L.G. 1960. A revision of the Melitainae genus Chlosyne and allied species (Lepidoptera: Nymphalidae). Trans. Roy. Entomol. Soc. Lond. 112: 381-467.

Hernández, V.H., I. Martínez \& S. Rodríguez. 1981. Lepidópteros en la Colección Entomológica de la Dirección General de Sanidad Vegetal. Parte I. Fitófilo 84: 15-17.

Hernández-Mejía, B.C. 2005. Composición y gremios alimentarios de mariposas diurnas de la superfamilia Papilionoidea (Insecta: Lepidoptera) en el municipio de Malinalco, Estado de México. Tesis de Biología. Universidad Autónoma del Estado de México, Toluca, México. 99 p.

Hernández-Mejía, B.C., J. Llorente-Bousquets, I. Vargas-Fernández \& A. Luis. 2008. Las mariposas (Hesperioidea y Papilionoidea) de Malinalco, Estado de México. Rev. Mex. Biodiv. 79: 117-130.

$\S$ Hoffmann, C.C. 1940. Catálogo Sistemático y Zoogeográfico de los Lepidópteros mexicanos. Primera Parte: Papilionoidea. An. Inst. Biol. UNAM (Zoología) 11: 639-739.

INEGI. 2004. Anuario Estadístico del Estado de México. Tomo I. Instituto Nacional de Estadística, Geografía e Informática. México D.F., México. 3-8 p.

Jenkins, D. 1983. Neotropical Nymphalidae I. Revision of Hamadryas. Bull. Allyn Mus. 81: 1-146.

Jenkins, D. 1986. Neotropical Nymphalidae V. Revision of Epiphile. Bull. Allyn Mus. 101: 1-70.

Kendall, R.O. \& W.W. McGuire. 1984. Some new and rare records of Lepidoptera found in Texas. Bull. Allyn Mus. 86: 1-50.

Llorente-Bousquets, J., A. Luis \& I. Vargas-Fernández. 2006. Apéndice general de Papilionoidea, Distribución Estatal y Provincias Biogeograficas, p. 733-792. En J.J. Morrone \& J. Llorente-Bousquets (eds.). Componentes bióticos principales de la Entomofauna 
Mexicana. Las Prensas de Ciencias, Facultad de Ciencias, UNAM. México D. F, México.

Luis, M.A., J. Llorente-Bousquets, I. Vargas-Fernández \& A.D. Warren. 2003. Biodiversity and Biogeography of mexican butterflies (Lepidoptera: Papilionoidea and Hesperioidea). Proc. Entomol. Soc. Washington. 105: 209-224.

Luis, M.A., J. Llorente-Bousquets \& I. Vargas-Fernández. 2005. Una megabase de datos de mariposas de México y la regionalización biogeográfica, p. 269294. En J. Llorente-Bousquets \& J.J. Morrone (eds.). Regionalización biogeográfica en Iberoamérica y tópicos afines: Primeras Jornadas Biogeográficas RIBES. Las Prensas Ciencias, Facultad de Ciencias, UNAM. México D.F., México.

Luna, I., J.J. Morrone \& D. Espinosa. 2007. Biodiversidad de la Faja Volcánica Transmexicana. UNAM. México D.F., México. 514 p.

Luna-Reyes, M. \& J. Llorente-Bousquets. 2004. Papilionoidea (Lepidoptera: Rhopalocera) de la Sierra Nevada, México. Acta Zool. Mex. (n.s.) 20: 79-102

Miller, L.D. 1974. Revision of the Euptychiini (Satyridae). 2. Cyllopsis R. Felder. Bull. Allyn Mus. 20: 1-98.

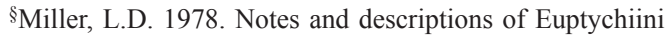
(Lepidoptera: Satyridae) from the Mexican region. Jour. Lep. Soc. 32: 75-85.

Miller, L.D. \& J. De la Maza. 1984. Notes on Cyllopsis, especially from Mexico, with description of a new species (Lepidoptera: Satyridae). Bull. Allyn Mus. 88: 1-7.

Rzedowski, J. 1978. La vegetación de México. Limusa, México D.F., México. 432 p.
Steinhauser, S.R. 1989. Taxonomic notes and descriptions of new taxa in the Neotropical Hesperiidae. Part. I. Pyrginae. Bull. Allyn Mus. 127: 1-70.

Vargas-Fernández, I., J. Llorente-Bousquets \& M.A. Luis. 1994. Listado lepidopterofaunístico de la Sierra de Atoyac de Álvarez en el Estado de Guerrero: Notas acerca de su distribución local y estacional (Rhopalocera: Papilionoidea). Folia Entomol. Mex. 86: 41-178

Vargas-Fernández, I., J. Llorente-Bousquets \& M.A. Luis. 1999. Distribución de los Papilionoidea (Lepidoptera: Rhopalocera) de la Sierra de Manantlán (250-1650 msnm) en los estados de Jalisco y Colima. Publ. Espec. Mus. Zool., UNAM, Fac. Ciencias, UNAM. 11: $1-153$.

Vázquez, L.G. 1951. Observaciones sobre piéridos mexicanos II. Phoebis sennae eubule y sus formas en México. An. Inst. Biol. UNAM (Zoología) 21: $417-$ 429.

Vilchis, M.A. 1992. Hipsometría, p. 26-27. En M.A. García, L. Mendoza, N. Pineda, S. Franco, M. Ocampo, A. Saker, R. Franco, J. Oscos \& S. Salazar (eds.). Atlas del Estado de México. Gobierno del Estado de México y Universidad Autónoma del Estado de México. Toluca, México.

Warren, A.D. 2000. Hesperioidea (Lepidoptera), p. 535580. En J. Llorente-Bousquets, E. González \& N. Papavero (eds.). Biodiversidad, Taxonomía y Biogeografía de Artrópodos de México: Hacia una Síntesis de su Conocimiento. Volumen II. Facultad de Ciencias, UNAM. México D. F., México.

$\S$ Referencia citada únicamente en el Apéndice 1. 


\section{APÉNDICE 1}

\section{HESPERIOIDEA Y PAPILIONOIDEA DEL ESTADO DE MÉXICO}

La lista a continuación sigue el arreglo filogenético propuesto por Warren (2000) y Llorente et al. (2006) e incluye el nombre de la superfamilia, la familia, la subfamilia y el nombre científico de la especie o subespecie, el municipio y la localidad de recolecta, el mes de vuelo (en número romano), la colección o cita en que se hace referencia (en paréntesis); se indica con un asterisco (*) si es un taxón endémico de México. Las claves de la colección y municipio se encuentran en los cuadros 1 y 2 , respectivamente.

\section{SUPERFAMILIA HESPERIOIDEA \\ FAMILIA HESPERIIDAE \\ SUBFAMILIA PYRRHOPYGINAE}

*Chalypyge chalybea (Scudder 1872). MALI: Malinalco VI-VII,IX (MCLB); X (MZFC); El Molino V,X, San Pedro Chichicasco V (CNIN: Hernández-Mejía 2005).

*Apyrrothrix araxes araxes (Hewitson 1867). MALI: Malinalco VI-XI (MCLB); TEN: Tenancingo VI (MCLB).

Pyrhopyge chloris Evans 1951. EM: San Nicolás (De la Maza 1987).

Elbella scylla (Ménétriés 1855). MALI: Colonia Juárez III (CNIN: Hernández-Mejía 2005).

Mysoria affinis (Herrich-Schäffer 1869). EM: San Nicolás Tolentino (De la Maza 1987).

\section{SUBFAMILIA PYRGINAE}

Phocides polybius lilea (Reakirt [1867]). MALI: El Platanar VII (CNIN: HernándezMejía 2005); VG: Villa Guerrero V (MZFC).

Udranomia kikkawai (Weeks 1906). VB: Valle de Bravo VIII (MCLB).

Epargyreus exadeus cruza Evans 1952. MALI: Chalma (CNIN: Guzmán 1976); Colonia Juárez V, El Platanar V, San Andrés Nicolás Bravo VI, San Pedro Chichicasco V (CNIN: Hernández-Mejía 2005).

Epargyreus sp. MALI: Colonia Juárez V (CNIN: Hernández-Mejía 2005).

Chioides albofasciatus (Hewitson 1867). MALI: Puente Caporal XII, Tepehuajes V (CNIN: Hernández-Mejía 2005).

Chioides zilpa (Butler 1872). MALI: Colonia Juárez III-IV, El Platanar II, La Angostura XI, San Pedro Chichicasco II (CNIN: Hernández-Mejía 2005).

Aguna asander asander (Hewitson 1867). MALI: Colonia Juárez V, El Molino IX, La Angostura X (CNIN: Hernández-Mejía 2005).

*Zestusa elwesi (Godman \& Salvin 1893). TEN: Tenancingo III (MCLB).

Codatractus sp. grupo arizonensis (Skinner 1905). MALI: El Platanar VII, Tepehuajes VIII (CNIN: Hernández-Mejía 2005).

Codatractus bryaxis (Hewitson 1867). MALI: Chalma (Guzmán 1976). 
*Codatractus uvydixa (Dyar 1914). MALI: Colonia Juárez VI, El Platanar V (CNIN: Hernández-Mejía 2005).

“Codatractus hyster” (Dyar 1916). MALI: Colonia Juárez V, San Pedro Chichicasco V (CNIN: Hernández-Mejía 2005).

Urbanus pronta Evans 1952. MALI: El Molino IV (CNIN: Hernández-Mejía 2005).

Urbanus prodicus Bell 1956. MALI: El Molino IV, Puente Caporal X (CNIN: Hernández-Mejía 2005).

Urbanus dorantes dorantes (Stoll [1790]). MALI: Malinalco III (MCLB); Colonia Juárez IV,X, El Molino IX, La Angostura VI,X, San Andrés Nicolás Bravo II (CNIN: Hernández-Mejía 2005).

Urbanus teleus (Hübner 1821). MALI: Chalma (Guzmán 1976); Puente Caporal X, San Andrés Nicolás Bravo VIII, Tepehuajes V (CNIN: Hernández-Mejía 2005).

Urbanus simplicius (Stoll 1790). MALI: Chalma (Guzmán 1976); Colonia Juárez III, El Molino V, La Angostura XI, San Andrés Nicolás Bravo VI, Tepehuajes V (CNIN: Hernández-Mejía 2005).

Urbanus procne (Plötz 1881). MALI: Colonia Juárez IV, El Molino IV, El Platanar IX, Jalmolonga IX, Puente Caporal XII (CNIN: Hernández-Mejía 2005).

Astraptes "fulgerator azul" (Reakirt [1867]). MALI: El Platanar IX, Puente Caporal X-XI (CNIN: Hernández-Mejía 2005).

Astraptes alector hopfferi (Plötz 1881). MALI: Colonia Juárez IV, San Andrés Nicolás Bravo IV (CNIN: Hernández-Mejía 2005).

Astraptes anaphus annetta Evans 1952. MALI: El Platanar XI (CNIN: HernándezMejía 2005).

Autochton cellus (Boisduval \& LeConte [1837]). ATZ: Atizapán VII-VIII (MCLB); MALI: Chalma VII (MCLB), (Guzmán 1976); Malinalco VII,IX-X (MCLB); Cerro “El Zapote” VII, Los Manantiales VII (CNIN: Hernández-Mejía 2005).

*Autochton pseudocellus (Coolidge \& Clemence [1910]). EM: Toluca, W de México $15 \mathrm{~km}$ VI (MZFC).

Autochton cincta (Plötz 1882). ZAC: Zacualpan II (MZFC).

Achalarus casica (Herrich-Schäffer 1869). MALI: Malinalco IX (MCLB); Chalma (Guzmán 1976).

Achalarus albociliatus albociliatus (Mabille 1877). MALI: Colonia Juárez III, La Angostura II, Puente Caporal X-XII, San Andrés Nicolás Bravo II,VI (CNIN: Hernández-Mejía 2005).

Achalarus toxeus (Plötz 1882). MALI: Colonia Juárez IV (CNIN: Hernández-Mejía et al. 2008).

Thorybes drusius (W.H. Edwards [1894]). EM: Almoloya IX (MCLB); MALI: San Pedro Chichicasco V (CNIN: Hernández-Mejía 2005).

Thorybes pylades (Scudder 1870). ATZ: Atizapán VII (MCLB).

Cabares potrillo potrillo (Lucas 1857). MALI: Colonia Juárez IV, San Andrés Nicolás Bravo X-XI (CNIN: Hernández-Mejía 2005). 
Celaenorrhinus fritzgaertneri (Bailey 1880). EM: San Nicolás Tolentino (De la Maza 1987); MALI: El Platanar V (CNIN: Hernández-Mejía 2005).

Spathilepia clonius (Cramer 1775). MALI: Colonia Juárez IV, Puente Caporal XI-XII (CNIN: Hernández-Mejía 2005).

Cogia hippalus hippalus (W.H. Edwards 1882). MALI: Malinalco IX (MCLB).

Cogia caicus moschus (W.H. Edwards 1882). EM: Almoloya IX (MCLB).

Cogia calchas (Herrich-Shäffer 1869). MALI: Chalma (Guzmán 1976); Puente Caporal II, Tepehuajes V (CNIN: Hernández-Mejía 2005).

Nisoniades ephora (Herrich-Shäffer 1870). MALI: Puente Caporal IX,XII (CNIN: Hernández-Mejía 2005).

Pellicia arina Evans 1953. MALI: El Platanar I (CNIN: Hernández-Mejía 2005).

Bolla orsines (Godman \& Salvin 1896). MALI: Cerro "El Zapote" VII, El Molino IX, El Platanar V, Los Manantiales VII (CNIN: Hernández-Mejía 2005).

*Bolla guerra Evans 1953. EM: Avándaro VI (MCLB).

Bolla eusebius (Plötz 1884). MALI: Malinalco XI (MCLB).

Staphylus vulgata (Möschler 1879). MALI: El Platanar VII (CNIN: HernándezMejía 2005).

*Staphylus tierra Evans 1953. MALI: Colonia Juárez IV, El Platanar VII,IX, Los Manantiales VII, Puente Caporal XII, San Pedro Chichicasco V (CNIN: Hernández-Mejía 2005).

Staphylus azteca (Scudder 1872). EM: Matlapa V (MCLB).

Staphylus iguala (R.C. Williams \& Bell 1940). MALI: Colonia Juárez IV, El Molino V, La Angostura VI, Puente Caporal XII, San Andrés Nicolás Bravo II (CNIN: Hernández-Mejía 2005).

Gorgythion begga pyralina (Möschler 1877). EM: Matlapa V (MCLB); MALI: Colonia Juárez IV-V, El Platanar I,IX (CNIN: Hernández-Mejía 2005).

Zera hyacinthinus hyacinthinus (Mabille 1877). EM: Almoloya IX (MCLB); Avándaro VI (MCLB); MALI: Malinalco VIII (MCLB).

Atarnes sallei (C. Felder \& R. Felder 1867). MALI: Colonia Juárez X, El Molino V (CNIN: Hernández-Mejía 2005).

Antigonus erosus (Hübner [1812]). MALI: Colonia Juárez IV, El Platanar VII,IX, Jalmolonga V, La Angostura II, Puente Caporal IX, San Andrés Nicolás Bravo VI (CNIN: Hernández-Mejía 2005).

*Antigonus emorsa (R. Felder 1869). MALI: Chalma (Guzmán 1976); Colonia Juárez III, El Platanar V, San Pedro Chichicasco V, Tepehuajes V,VIII (CNIN: Hernández-Mejía 2005).

*Antigonus funebris (R. Felder 1869). MALI: Chalma (Guzmán 1976); Jalmolonga V, San Pedro Chichicasco V (CNIN: Hernández-Mejía 2005).

Zopyrion sandace Godman \& Salvin 1896. MALI: Colonia Juárez IV,X, El Platanar IX (CNIN: Hernández-Mejía 2005).

Achlyodes pallida (R. Felder 1869). MALI: La Angostura X, Puente Caporal XI (CNIN: Hernández-Mejía 2005). 
Eantis tamenund (W.H. Edwards 1871). MALI: Puente Caporal X (CNIN: Hernández-Mejía 2005).

*Doberes hewitsonius (Reakirt [1867]). MALI: Camino viejo a Ocuilan VII (CNIN: Hernández-Mejía 2005).

Ebrietas anacreon anacreon (Staudinger 1876). MALI: El Platanar IX, San Andrés Nicolás Bravo II (CNIN: Hernández-Mejía 2005).

Cycloglypha thrasibulus (Fabricius 1793). EM: San Nicolás Tolentino (De la Maza 1987).

Chiomara georgina georgina (Reakirt 1868). MALI: Colonia Juárez IV, El Molino V, El Platanar I,IX, Puente Caporal II,XII, Tepehuajes VIII (CNIN: HernándezMejía 2005).

Chiomara mithrax (Möschler 1879). MALI: El Platanar I (CNIN: Hernández-Mejía 2005).

Gesta invisus (Butler \& H. Druce 1872). MALI: Colonia Juárez III, El Platanar I,IX, San Andrés Nicolás Bravo IV (CNIN: Hernández-Mejía 2005).

Erynnis tristis tristis (Boisduval 1852). TEX: Chapingo (INIA: Domínguez \& Carrillo 1976).

Erynnis funeralis (Scudder \& Burgess 1870). MALI: Colonia Juárez X, El Platanar I, Tepehuajes VIII (CNIN: Hernández-Mejía 2005).

Pyrgus communis (Grote 1872). MALI: Chalma (Guzmán 1976); Cerro "El Zapote” VII (CNIN: Hernández-Mejía 2005); TEM: Parque Universitario "Las Orquídeas" IX (Aguilera-Ríos \& Hernández-Mejía, en prep.); TEX: Chapingo (COEE: Gibson \& Carrillo 1959).

Pyrgus albescens Plötz 1884. MALI: Cerro "El Zapote" VII, Colonia Juárez III, El Molino IV, Jalmolonga V, La Angostura VI, San Andrés Nicolás Bravo VI (CNIN: Hernández-Mejía 2005).

Pyrgus oileus (Linnaeus 1767). MALI: Chalma VII (MZFC); Camino viejo a Ocuilan VII, Cerro "El Zapote" VII, Colonia Juárez III-IV, El Molino V, El Platanar VII, Jalmolonga V, La Angostura II,VI, Los Manantiales V, Puente Caporal IX, Tepehuajes V,VIII (CNIN: Hernández-Mejía 2005); ZAC: Puerto Obscuro I,II (MZFC).

Heliopetes macaira macaira (Reakirt [1867]). MALI: Chalma (Guzmán 1976); Cerro "El Zapote" VII, El Platanar VII, Jalmolonga V,IX (CNIN: HernándezMejía 2005).

Pholisora mejicanus (Reakirt [1867]). MALI: Chalma (Guzmán 1976); Camino viejo a Ocuilan VII, El Molino V, Jalmolonga V (CNIN: Hernández-Mejía 2005).

\section{SUBFAMILIA CYCLOPEDINAE}

*Piruna brunnea (Scudder 1872). MALI: Cerro "El Zapote" VII, El Molino IX (CNIN: Hernández-Mejía 2005).

*Dalla dividuum (Dyar 1913). MALI: El Molino V (CNIN: Hernández-Mejía 2005). 


\section{SUBFAMILIA HESPERIINAE}

*Synapte syraces (Godman 1901). MALI: Colonia Juárez IV (CNIN: HernándezMejía et al. 2008).

Callimormus saturnus (Herrich-Schäffer 1869). MALI: Cerro "El Zapote" VII, Colonia Juárez III-IV, El Molino IX, La Angostura II (CNIN: Hernández-Mejía 2005).

Nastra julia (H.A. Freeman 1945). MALI: El Platanar IX, Jalmolonga V,IX (CNIN: Hernández-Mejía 2005).

Cymaenes trebius (Mabille 1891). MALI: Colonia Juárez IV (CNIN: HernándezMejía et al. 2008).

Vehilius inca (Scudder 1872). MALI: Colonia Juárez IV (CNIN: Hernández-Mejía et al. 2008).

Lerema accius (J.E. Smith 1797). MALI: Colonia Juárez IV, El Platanar IX (CNIN: Hernández-Mejía 2005).

Vettius fantasos (Cramer 1780). MALI: Colonia Juárez III-IV, La Angostura X (CNIN: Hernández-Mejía 2005).

Perichares philetes adela (Hewitson [1867]). MALI: Colonia Juárez IV (CNIN: Hernández-Mejía et al. 2008).

Quinta cannae (Herrich-Shäffer 1869). MALI: El Molino IV, Puente Caporal X (CNIN: Hernández-Mejía 2005).

Rhinthon osca (Plötz 1882). MALI: Colonia Juárez V (CNIN: Hernández-Mejía 2005).

Conga chydaea (Butler 1877). MALI: Colonia Juárez IV (CNIN: Hernández-Mejía et al. en prensa).

Ancyloxypha arene (W.H. Edwards 1871). MALI: Colonia Juárez III,V, El Molino V,IX, Jalmolonga V, Puente Caporal IX (CNIN: Hernández-Mejía 2005); TEX: Chapingo (INIA: Domínguez \& Carrillo 1976).

Copaeodes minima (W.H. Edwards 1870). MALI: Chalma (Guzmán 1976); Colonia Juárez III-IV, El Molino IV, El Platanar IX, Jalmolonga V (CNIN: HernándezMejía 2005); ZAC: Zacualpan IX,XI (MZFC).

Polites vibex praeceps (Scudder 1872). MALI: Colonia Juárez IV, Tepehuajes V, San Andrés Nicolás Bravo II (CNIN: Hernández-Mejía 2005).

Wallengrenia otho otho (J.E. Smith 1797). MALI: Puente Caporal XII (CNIN: Hernández-Mejía 2005).

Pompeius pompeius (Latreille [1824]). MALI: Colonia Juárez III, El Molino IV, La Angostura X, Puente Caporal X (CNIN: Hernández-Mejía 2005).

Poanes zabulon (Boisduval \& LeConte [1837]). ZAC: Tres Cruces de Mamatla (MZFC).

*Poanes monticola (Godman 1900). EM: Toluca, W de México 15, km 18 VI (MZFC).

Quasimellana eulogius (Plötz 1883). MALI: El Platanar I (CNIN: Hernández-Mejía 2005). 
Amblyscirtes sp. MALI: El Platanar I,VII, San Pedro Chichicasco II (CNIN: Hernández-Mejía 2005).

Panoquina ocola ocola (W.H. Edwards 1863). MALI: Puente Caporal IX, San Andrés Nicolás Bravo II (CNIN: Hernández-Mejía 2005).

Thespieus dalman (Latreille [1824]). MALI: Colonia Juárez V, San Pedro Chichicasco V (CNIN: Hernández-Mejía 2005).

\section{SUPERFAMILIA PAPILIONOIDEA}

FAMILIA PAPILIONIDAE

SUBFAMILIA BARONIINAE

*Baronia brevicornis brevicornis Salvin 1893. MALI: El Platanar V (CNIN: Hernández-Mejía 2005).

SUBFAMILIA PAPILIONINAE

*Protographium epidaus fenochionis (Salvin \& Godman 1868). EM: San Nicolás (De la Maza \& De la Maza 1978); MALI: San Pedro Chichicasco V, Tepehuajes V (CNIN: Hernández-Mejía 2005).

*Mimoides thymbraeus aconophos (Gray, [1853]). CHIA: Tenancingo IX (MZFC); EM: San Nicolás (De la Maza \& De la Maza 1978); MALI: Chalma (Guzmán 1976); El Molino IV, El Platanar V (CNIN: Hernández-Mejía 2005); VG: Villa Guerrero V (MZFC).

Battus laodamas iopas (Godman \& Salvin 1897). EM: San Nicolás III (CNIN); San Nicolás Tolentino (De la Maza \& De la Maza 1978); ST: Santo Tomás de los Plátanos VI (CNIN).

Battus philenor philenor (Linnaeus 1771). EM: Cerro de Chiconautla (De la Maza \& Turrent 1978); San Nicolás (De la Maza \& De la Maza 1978); Sierra Nevada (FES-Z: Luna-Reyes \& Llorente-Bousquets 2004); MALI: Tepehuajes VIII (CNIN: Hernández-Mejía 2005); TEO: Teotihuacán (De la Maza \& Turrent 1978); TEX: Texcoco X (MZFC).

Battus polydamas polydamas (Linnaeus 1758). EM: San Nicolás Tolentino (De la Maza \& De la Maza 1978); MALI: El Platanar VII, San Andrés Nicolás Bravo XI (CNIN: Hernández-Mejía 2005).

*Parides alopius (Godman \& Salvin 1890). CHIA: Tenancingo VI (MZFC); MALI: Malinalco VI,VIII (AME).

*Parides erithalion trichopus (Rothschild \& Jordan 1906). EM: La Joya IV (CNIN); San Nicolás XII (De la Maza 1980); San Nicolás Tolentino VI-XII (De la Maza \& De la Maza 1978); MALI: Colonia Juárez VI, San Andrés Nicolás Bravo II (CNIN: Hernández-Mejía 2005); TEM: Parque Universitario "Las Orquídeas" VI,X (Aguilera-Ríos y Hernández-Mejía, en prep.).

Parides montezuma (Westwood 1842). EM: San Nicolás III (CNIN); San Nicolás Tolentino (De la Maza \& De la Maza 1978); MALI: El Platanar V, San Andrés Nicolás Bravo VI,VIII (CNIN: Hernández-Mejía 2005).

Parides photinus (Doubleday 1844). EM: San Nicolás Tolentino (De la Maza \& De la Maza 1978); Sierra Nevada (FES-Z: Luna-Reyes \& Llorente-Bousquets 2004); MALI: Malinalco IX (AME); Puente Caporal XI (CNIN: Hernández-Mejía 
2005); TEJ: El Estanco, Sierra de Nanchititla (Barrera \& Díaz-Batres 1977); TEM: Parque Universitario "Las Orquídeas" V (Aguilera- Ríos \& HernándezMejía, en prep.).

Heraclides anchisiades idaeus (Fabricius 1793). EM: San Nicolás (De la Maza \& De la Maza 1978).

Heraclides cresphontes (Cramer 1777). EM: San Nicolás Tolentino (De la Maza \& De la Maza 1978); MALI: Colonia Juárez III (CNIN: Hernández-Mejía 2005).

Heraclides ornythion ornythion (Boisduval 1836). MALI: San Pedro Chichicasco V (CNIN: Hernández-Mejía 2005).

Heraclides rogeri pharnaces (Doubleday 1846). ALQ: Almoloya de Alquisiras V (MZFC); EM: San Nicolás (De la Maza \& De la Maza 1978); Sierra Nevada (FES-Z: Luna-Reyes \& Llorente-Bousquets 2004); MALI: Malinalco VII-VIII (AME); Colonia Juárez III (CNIN: Hernández-Mejía 2005); ST: Santo Tomás de los Plátanos III (CNIN).

Heraclides thoas autocles (Rothschild \& Jordan 1906). EM: San Nicolás (De la Maza \& De la Maza 1978); MALI: Malinalco VIII (AME); La Angostura VI (CNIN: Hernández-Mejía 2005).

Papilio polyxenes asterius Stoll 1782. AMEC: Amecameca (Godman \& Salvin 1878-1901); EM: Nepantla VIII (USNM); San Nicolás Tolentino (De la Maza \& De la Maza 1978); Sierra Nevada (FES-Z: Luna-Reyes \& Llorente-Bousquets 2004); Tetcutzingo X (MZFC); MALI: El Platanar XI, Tepehuajes V (CNIN: Hernández-Mejía 2005); TEJ: El Estanco, Sierra de Nanchititla (Barrera \& DíazBatres 1977); TEM: Parque Universitario “Las Orquídeas” II,XI (Aguilera- Ríos \& Hernández-Mejía, en prep.); TEX: XI (MZFC).

*Pterourus garamas garamas (Geyer [1829]). CHIA: Tenancingo VIII (MZFC); EM: El Polvorín I (MZFC); Popocatépetl 1 Park IX (CMNH); San Nicolás Tolentino (De la Maza \& De la Maza 1978); Sierra Nevada (FES-Z: Luna-Reyes \& Llorente-Bousquets 2004); MALI: Malinalco VI (CNIN), VI-IX,XI (AME), VIII (USNM), VI (CAS); Chalma (Guzmán 1976); El Platanar IX (CNIN: Hernández-Mejía 2005); TEM: Parque Universitario "Las Orquídeas" IV,XII (Aguilera-Ríos \& Hernández-Mejía, en prep.); TUL: Tultepec I,XI (MZFC); VG: Villa Guerrero V (MZFC); ZAC: Tres Cruces de Mamatla II,XII (MZFC).

*Pterourus menatius morelius (Rothschild \& Jordan 1906). EM: Moaglos VIII (AME); San Nicolás IX-X (CNIN); San Nicolás Tolentino (De la Maza \& De la Maza 1978); MALI: Malinalco (Beutelspacher \& Howe 1984), VI (CNIN), VI-X (AME), VIII,XI (MZFC), (Adams 1983); Colonia Juárez X, El Molino V (CNIN: Hernández-Mejía 2005); VG: Villa Guerrero V-VI (MZFC).

Pterourus multicaudata multicaudata (W.F. Kirby 1884). AZ: Atizapán de Zaragoza IV (MZFC); CHIA: Tenancingo VIII (MZFC); EM: Cascada Los Diamantes, San Rafael III-IV (MZFC), III (Barrera \& Romero 1986); San Nicolás Tolentino (De la Maza \& De la Maza 1978); Sierra Nevada (FES-Z: Luna-Reyes \& LlorenteBousquets 2004); MALI: Malinalco VI (AME); Chalma (Guzmán 1976); El Platanar V,VII (CNIN: Hernández-Mejía 2005); NAU: Naucalpan IV (AME); TEM: Parque Universitario "Las Orquídeas" III,XI (Aguilera-Ríos y HernándezMejía, en prep.); TEX: Chapingo (COEE: Gibson \& Carrillo 1959); Toluca 
(De la Maza 1987); El Cerrillo Piedras Blancas V-VII; VG: Villa Guerrero VI (MZFC).

FAMILIA PIERIDAE

\section{SUBFAMILIA DISMORPHIINAE}

*Enantia mazai diazi Llorente 1984. EM: Sierra Nevada (FES-Z: Luna-Reyes \& Llorente-Bousquets 2004); MALI: Malinalco VIII (AME); Chalma X (CNIN); Puente Caporal XI-XII (CNIN: Hernández-Mejía 2005); TEJ: El Reparo, Sierra de Nanchititla VII (MZFC); TEM: Parque Universitario "Las Orquídeas” III,X (Aguilera-Ríos y Hernández-Mejía, en prep.).

\section{SUBFAMILIA COLIADINAE}

Colias eurytheme Boisduval 1852. AZ: Atizapán de Zaragoza V,IX (CNIN), VIII (AME); CHIA: Tenancingo VIII (MZFC); EM: Cascada Los Diamantes, San Rafael IV (MZFC), (Barrera \& Romero 1986); Llano Grande X (CNIN); San Bartolo X (CNIN); (AMNH); Sierra Nevada (FES-Z: Luna-Reyes \& LlorenteBousquets 2004); TEX: Chapingo XI (MZFC), (COEE: Gibson \& Carrillo 1959), III,IX (CNIN); TOL: Toluca (De la Maza 1987); El Cerrillo Piedras Blancas X.

Zerene cesonia cesonia (Stoll 1790). ATZ: Ciudad A. López Mateos VIII (CNIN); AZ: Atizapán de Zaragoza VII (CNIN), VIII (AME); EM: carretera MéxicoToluca, km 18 VIII (CNIN); Cascada Los Diamantes, San Rafael II-III,VII,XI (MZFC), (Barrera \& Romero 1986); Llano Grande X (CNIN); Sierra Nevada (FES-Z: Luna-Reyes \& Llorente-Bousquets 2004); MALI: Malinalco III (AME); Colonia Juárez X, El Molino IV, El Platanar VII, La Angostura XI, Tepehuajes VIII (CNIN: Hernández-Mejía 2005); OA: Ocuilan-Cuernavaca, km 14 I (MZFC); TEJ: Cañada Bosque Encantado (Barrera \& Díaz-Batres 1977); TEM: Parque Universitario "Las Orquídeas” VIII,X (Aguilera-Ríos y Hernández-Mejía, en prep.); TEX: Chapingo (COEE: Gibson \& Carrillo 1959); TOL: El Cerrillo Piedras Blancas VI; TON: camino al Cerro de la Cruz XII (CNIN).

Anteos clorinde (Godart [1824]). EM: Cascada Los Diamantes, San Rafael III-IV (MZFC), (Barrera \& Romero 1986); Sierra Nevada (FES-Z: Luna-Reyes \& Llorente-Bousquets 2004); MALI: Malinalco VIII (AME); Chalma (Guzmán 1976); El Molino V (CNIN: Hernández-Mejía 2005); TEX: Chapingo (INIA: Domínguez \& Carrillo 1976); TOL: Toluca (De la Maza 1987).

Anteos maerula (Fabricius 1775). EM: Cascada Los Diamantes, San Rafael IV (MZFC); Sierra Nevada (FES-Z: Luna-Reyes \& Llorente-Bousquets 2004); MALI: Malinalco VIII,X (AME); Chalma (Guzmán 1976); Colonia Juárez III, El Platanar XI (CNIN: Hernández-Mejía 2005); TEM: Parque Universitario "Las Orquídeas” I,VI,XII (Aguilera-Ríos y Hernández-Mejía, en prep.); TOL: Toluca (De la Maza 1987); El Cerrillo Piedras Blancas VI.

Phoebis agarithe agarithe (Boisduval 1836). EM: Sierra Nevada (FES-Z: LunaReyes \& Llorente-Bousquets 2004); MALI: Malinalco VIII (AME); Colonia Juárez III-V, El Platanar V, La Angostura X, San Andrés Nicolás Bravo X (CNIN: Hernández-Mejía 2005); TEJ: El Estanco, Sierra de Nanchititla (Barrera \& DíazBatres 1977).

Phoebis argante ssp. n. MALI: Tepehuajes V, San Pedro Chichicasco V (CNIN: Hernández-Mejía 2005); OA: Ocuilan-Cuernavaca, km 14 III (MZFC). 
Phoebis neocypris virgo (Butler 1870). EM: Sierra Nevada (FES-Z: Luna-Reyes \& Llorente-Bousquets 2004); MALI: Malinalco VIII (CNIN), III,VI-VIII,X (AME), (CTE), (Vázquez 1951); Chalma (Guzmán 1976), VII (AME); Colonia Juárez III, Tepehuajes V (CNIN: Hernández-Mejía 2005); TEM: Parque Universitario "Las Orquídeas" IV,X (Aguilera-Ríos y Hernández-Mejía, en prep.); OA: OcuilanCuernavaca, km 14 III (MZFC); ZAC: Tres Cruces de Mamatla XII (MZFC).

Phoebis philea philea (Linnaeus 1763). EM: Cascada Los Diamantes, San Rafael I (MZFC), (Barrera \& Romero 1986); Sierra Nevada (FES-Z: Luna-Reyes \& Llorente-Bousquets 2004); MALI: Malinalco VIII (AME); Colonia Juárez V, San Pedro Chichicasco V (CNIN: Hernández-Mejía 2005); TEM: Parque Universitario “Las Orquídeas” I,VI,XII (Aguilera- Ríos \& Hernández-Mejía, en prep.); OA: Ocuilan-Cuernavaca, km 14 III (MZFC).

Phoebis sennae marcellina (Cramer 1777). AZ: Atizapán de Zaragoza VI (MZFC); EM: Sierra Nevada (FES-Z: Luna-Reyes \& Llorente-Bousquets 2004); MALI: Chalma (Guzmán 1976); Camino viejo a Ocuilan VII, Colonia Juárez III-IV, El Platanar I,V, Jalmolonga V, La Angostura VI, San Pedro Chichicasco IX (CNIN: Hernández-Mejía 2005); OA: Ocuilan-Cuernavaca, km 14 I (MZFC); TEJ: El Estanco, Sierra de Nanchititla (Barrera \& Díaz-Batres 1977); TEM: Parque Universitario "Las Orquídeas” XI (Aguilera-Ríos y Hernández-Mejía, en prep.).

Rhabdodryas trite ssp. n. MALI: Chalma (Guzmán 1976); Malinalco V-VIII,XI (AME); El Molino V (CNIN: Hernández-Mejía 2005).

Aphrissa statira statira (Cramer 1777). TEX: Chapingo (COEE: Gibson \& Carrilo 1959).

Abaeis nicippe (Cramer 1779). EM: Cascada Los Diamantes, San Rafael (Barrera \& Romero 1986); Sierra Nevada (FES-Z: Luna-Reyes \& Llorente-Bousquets 2004); MALI: El Platanar V, San Andrés Nicolás Bravo VIII,X (CNIN: HernándezMejía 2005); TEJ: Cañada Bosque Encantado (Barrera \& Díaz-Batres 1977).

Pyrisitia dina westwoodi (Boisduval 1836). AZ: Atizapán de Zaragoza VI (AME); MALI: Colonia Juárez III, El Platanar V,VII,IX, La Angostura VI, Puente Caporal IX, San Pedro Chichicasco V, Tepehuajes V,VIII (CNIN: HernándezMejía 2005); TEM: Parque Universitario “Las Orquídeas” IV,X (Aguilera-Ríos y Hernández-Mejía, en prep.).

Pyrisitia nise nelphe (R. Felder 1869). EM: Popocatépetl VI (USNM); Sierra Nevada (FES-Z: Luna-Reyes \& Llorente-Bousquets 2004), Zirimicuaro VI (MZFC); MALI: Colonia Juárez VI, El Platanar IX, Puente Caporal XII, San Andrés Nicolás Bravo VIII,XI, Tepehuajes VIII, (CNIN: Hernández-Mejía 2005); ZAC: Tres Cruces de Mamatla II (MZFC).

Pyrisitia proterpia (Fabricius 1775). EM: Cascada Los Diamantes, San Rafael XII (MZFC), (Barrera \& Romero 1986); Nuevo Morelos XI (AME); Río Frío, 2 km W VIII (UCB); MALI: Chalma (Guzmán 1976), III (CNIN); Camino viejo a Ocuilan VII, Cerro "El Zapote” VII, Colonia Juárez III, El Platanar V,VII, La Angostura VI, Puente Caporal X, Tepehuajes VIII (CNIN: Hernández-Mejía 2005); OA: Ocuilan-Cuernavaca, km 14 III (MZFC); TEJ: Cañada Bosque Encantado, Cañada La Canoa, $2.5 \mathrm{~km}$ del Reparo (Barrera \& Díaz-Batres 1977); TEM: Parque Universitario "Las Orquídeas" VIII-X (Aguilera-Ríos y Hernández-Mejía, en prep.); TOL: El Cerrillo Piedras Blancas V-VIII; TON: Camino al Cerro de la Cruz XII (CNIN). 
Eurema arbela boisduvaliana (C. Felder \& R. Felder 1865). EM: Sierra Nevada (FES-Z: Luna-Reyes \& Llorente-Bousquets 2004); Zirimicuaro III (MZFC); MALI: Malinalco (AME); Tepehuajes VIII (CNIN: Hernández-Mejía 2005); OA: Ocuilan-Cuernavaca, km 14 I (MZFC).

Eurema daira sidonia (R. Felder 1869). EM: Cascada Los Diamantes, San Rafael (Barrera \& Romero 1986); Los Reyes, 2 mi SW VII (UCB); Sierra Nevada (FESZ: Luna-Reyes \& Llorente-Bousquets 2004); IXS: Ixtapan de la Sal VII (AME); MALI: Chalma (Guzmán 1976); Malinalco VIII (AME); Colonia Juárez VI, El Molino IV, El Platanar VII, Jalmolonga V, San Andrés Nicolás Bravo VI,VIII, Tepehuajes V (CNIN: Hernández-Mejía 2005); TEJ: Cañada Bosque Encantado (Barrera \& Díaz-Batres 1977); Cañada La Canoa, 2.5 km del Reparo (Barrera \& Díaz-Batres 1977); El Estanco, Sierra de Nanchititla (Barrera \& Díaz-Batres 1977); TON: Camino al Cerro de la Cruz XII (CNIN); ZUM: Zumpango VII (CNIN).

Eurema mexicana mexicana (Boisduval 1836). AZ: Atizapán de Zaragoza VI (MZFC); CHIA: Tenancingo VII (CNIN); EM: Cascada Los Diamantes, San Rafael II (MZFC), (Barrera \& Romero 1986); Sierra Nevada (FES-Z: LunaReyes \& Llorente-Bousquets 2004); MALI: Malinalco VII (AME); Cerro "El Zapote" VII, Puente Caporal XII (CNIN: Hernández-Mejía 2005); OA: OcuilanCuernavaca, km 14 I,III (MZFC); TEM: Parque Universitario "Las Orquídeas" II,XII (Aguilera-Ríos y Hernández-Mejía, en prep.); VB: Valle de Bravo (AME).

Eurema salome jamapa (Reakirt 1866). EM: Cascada Los Diamantes, San Rafael IV,XI (MZFC), (Barrera \& Romero 1986); Sierra Nevada (FES-Z: Luna-Reyes \& Llorente-Bousquets 2004); MAL: Cerro "El Zapote" VII, Puente Caporal IX, San Andrés Nicolás Bravo II,VIII (CNIN: Hernández-Mejía 2005); OA: OcuilanCuernavaca, km 14 I (MZFC); TEJ: Cañada La Canoa, $2.5 \mathrm{~km}$ del Reparo (Barrera \& Díaz-Batres 1977); TEM: Parque Universitario "Las Orquídeas” IX-X (Aguilera-Ríos y Hernández-Mejía, en prep.).

Nathalis iole Boisduval 1836. AZ: Atizapán de Zaragoza VII (AME); EM: Cascada Los Diamantes, San Rafael (Barrera \& Romero 1986); El Polvorín I (MZFC); Ixtapantongo X (CNIN); San Bartolo X (CNIN); Sierra Nevada (FES-Z: LunaReyes \& Llorente-Bousquets 2004); IXS: Ixtapan de la Sal VII (CNIN); MALI: Chalma (Guzmán 1976); El Molino IV, Jalmolonga V, Tepehuajes V (CNIN: Hernández-Mejía 2005); ST: Santo Tomás de los Plátanos III (CNIN); TEX: Chapingo (Gibson \& Carrillo 1959); TOL: El Cerrillo Piedras Blancas VI-VII; TON: Camino al Cerro de la Cruz (CNIN); TULN: Presa de Guadalupe (DGSV: Hernández et al. 1981); TUL: VII (MZFC); ZAC: Puerto Obscuro XI (MZFC).

\section{SUBFAMILIA PIERINAE}

*Anthocharis limonea (Butler 1871). AZ: Atizapán de Zaragoza VII (MZFC), (De la Maza 1987), VII-VIII (CNIN), II,VII (AME), (DGSV: Hernández et al. 1981), (Beutelspacher 1976).

Hesperocharis costaricensis pasion (Reakirt [1867]). EM: Sierra Nevada (FES-Z: Luna-Reyes \& Llorente-Bousquets 2004); MALI: Puente Caporal IX (CNIN: Hernández-Mejía, 2005); OA: Ocuilan-Cuernavaca, km 14 III (MZFC). 
*Hesperocharis graphites avivolans (Butler 1865). AMEC: Amecameca (USNM), (Godman \& Salvin 1878-1901); ATL: Atlauta IV (CNIN); EM: Cascada Los Diamantes, San Rafael II,IV (MZFC), (Barrera \& Romero 1986); Sierra Nevada (FES-Z: Luna-Reyes \& Llorente-Bousquets 2004); OA: Mexicapan XI,XII (MZFC); Ocuilan-Cuernavaca, km 14 I,III,VI (MZFC); VB: Valle de Bravo I (AME); ZAC: Puerto Obscuro, 200 mi NE IX (MZFC).

*Eucheira socialis socialis Westwood 1834. AMEC: Amecameca (Godman \& Salvin 1878-1901); EM: carretera Villa Victoria-Valle de Bravo, km 22 I-II,IV-V (CNIN), (Beutelspacher 1983), (De la Maza 1987), V (AME); El Polvorín III (MZFC); Oxtoltiplan 1 km N de San Francisco III (MZFC); VB: Valle de Bravo IV (MZFC), V (AME); ZAC: Zacualpan (CNIN), IV (AMNH), V (LACM), (Beutelspacher 1983).

*Catasticta nimbice nimbice (Boisduval 1836). EM: Cascada Los Diamantes, San Rafael II,V (Barrera \& Romero 1986); El Polvorín I; San Dieguito VIII; Santa Mónica III (MZFC); Sierra Nevada (FES-Z: Luna-Reyes \& Llorente-Bousquets 2004); MALI: Puente Caporal X (CNIN: Hernández-Mejía 2005); OA: OcuilanCuernavaca, km 13 I,III,VI (MZFC); ST: Santo Tomás de los Plátanos IX (CNIN); TOL: El Cerrillo Piedras Blancas VI; VG: Villa Guerrero V (MZFC); ZAC: Puerto Obscuro II (MZFC); Tres Cruces de Mamatla II (MZFC).

*Catasticta teutila teutila (Doubleday 1847). AMEC: Amecameca (Godman \& Salvin 1878-1901), IX (CAS); ATL: Atlauta (DGSV: Hernández et al. 1981); EM: Cascada Los Diamantes, San Rafael X (MZFC), XI-XII (Barrera \& Romero 1986); Llano Grande X (CNIN); San Dieguito I (MZFC); Sierra Nevada (FES-Z: Luna-Reyes \& Llorente-Bousquets 2004); Tlalmanalco VI (AME).

Melete lycimnia isandra (Boisduval 1836). EM: Ixtapantongo (De la Maza 1987).

Glutophrissa drusilla tenuis (Lamas 1981). EM: San Nicolás XI (CNIN); MALI: Malinalco VI (AME); La Angostura XI (CNIN: Hernández-Mejía 2005); NAU: Naucalpan VI (MZFC); OA: Ocuilan-Cuernavaca, km 14 I (MZFC); ST: Santo Tomás de los Plátanos V (CNIN), (De la Maza 1987); VB: Colorines (De la Maza 1987).

Pieris rapae rapae (Linnaeus 1758). TEX: Chapingo (INIA: Domínguez \& Carrillo 1976).

Pontia protodice (Boisduval \& LeConte [1830]). AZ: Atizapán de Zaragoza VIII (AME); EM: Cascada Los Diamantes, San Rafael I,IV,XII (MZFC), (Barrera \& Romero 1986); Sierra Nevada (FES-Z: Luna-Reyes \& Llorente-Bousquets 2004); MALI: Malinalco IX (AME); OA: Ocuilan-Cuernavaca, km 14 III (MZFC); TEX: Texcoco VII (MZFC); Chapingo VIII,X (MZFC), (COEE: Gibson \& Carrillo 1959); ZUM: Zumpango VIII (CNIN).

Leptophobia aripa elodia (Boisduval 1836). CHIA: Tenancingo VIII (MZFC); EM: carretera México-Toluca, km 18 XI (CNIN); Cascada Los Diamantes, San Rafael I,IV,XI (MZFC), (Barrera \& Romero 1986); Sierra Nevada (FES-Z: Luna-Reyes \& Llorente-Bousquets 2004); MALI: Colonia Juárez III, Puente Caporal X, San Andrés Nicolás Bravo II,VIII, Tepehuajes VIII (CNIN: Hernández-Mejía 2005); OA: Ocuilan-Cuernavaca, km 14 I,III (MZFC); TEM: Parque Universitario "Las Orquídeas" VI-VII (Aguilera-Ríos y Hernández-Mejía, en prep.); TEX: Chapingo (INIA: Domínguez \& Carrillo 1976), (COEE: Gibson \& Carrillo 1959); TOL: El Cerrillo Piedras Blancas VIII; TON: Camino al Cerro de la Cruz (CNIN). 
Pieriballia viardi (Boisduval 1836). MALI: Colonia Juárez III (CNIN: HernándezMejía 2005).

Ascia monuste monuste (Linnaeus 1764). EM: Sierra Nevada (FES-Z: Luna-Reyes \& Llorente-Bousquets 2004); MALI: Colonia Juárez III, El Platanar I, Puente Caporal XII, (CNIN: Hernández-Mejía 2005); TON: Camino al Cerro de La Cruz VII (CNIN).

Ganyra josephina josepha (Salvin \& Godman 1868). MALI: San Andrés Nicolás Bravo VI (CNIN: Hernández-Mejía 2005).

\section{FAMILIA LYCAENIDAE}

SUBFAMILIA THECLINAE

Brangas neora (Hewitson 1867). MALI: Tepehuajes VIII (CNIN: Hernández-Mejía 2005).

Evenus regalis (Cramer 1775). EM: San Nicolás Tolentino (De la Maza 1987).

Atlides carpasia (Hewitson 1868). TEM: El Polvorín I (MZFC); VG: Villa Guerrero $\mathrm{V}(\mathrm{MZFC})$.

Pseudolycaena damo (H. Druce 1875). MALI: La Angostura VI (CNIN: HernándezMejía 2005).

Micandra cyda (Godman \& Salvin 1887). AMEC: Amecameca (Godman \& Salvin 1878-1901); EM: carretera Villa Victoria-Valle de Bravo, km 22 IV (CNIN); Cascada Los Diamantes, San Rafael II,V,X (MZFC), (Barrera \& Romero 1986); Las Manzanas XI (CNIN); Popocatépetl VI-VII (USNM); Sierra Nevada (FESZ: Luna-Reyes \& Llorente-Bousquets 2004); OA: Mexicapan XII (MZFC); Ocuilan-Cuernavaca, km 14 VI (MZFC).

Themecla paron (Godman \& Salvin 1887). TEM: El Polvorín I (MZFC).

Thereus orasus (Godman \& Salvin 1887). OA: Ocuilan-Cuernavaca, km 14 I (MZFC).

Rekoa palegon (Cramer 1780). ALQ: Almoloya de Alquisiras (CNIN); ZAC: Tres Cruces de Mamatla II (MZFC); Puerto Obscuro IX (MZFC).

Rekoa marius (Lucas 1857). MALI: La Angostura VI, San Andrés Nicolás Bravo VI (CNIN: Hernández-Mejía 2005).

Arawacus jada (Hewitson 1867). EM: Acatenango, Chimatenango VII,IX (MZFC); Sierra Nevada (FES-Z: Luna-Reyes \& Llorente-Bousquets 2004); MALI: Chalma (Guzmán 1976); Jalmolonga V (CNIN: Hernández-Mejía 2005); TEM: Parque Universitario "Las Orquídeas" III,VIII (Aguilera-Ríos y HernándezMejía, en prep.); VG: Villa Guerrero IX (CNIN); ZAC: Tres Cruces de Mamatla II (MZFC).

Contrafacia bassania (Hewitson 1868). OA: Ocuilan-Cuernavaca, km 14 I,III (MZFC); TEM: El Polvorín I (MZFC).

Ocaria ocrisia (Hewitson 1868). MALI: Puente Caporal XII (CNIN: HernándezMejía 2005); OA: Ocuilan-Cuernavaca, km 14 I (MZFC).

Chlorostrymon simaethis (Drury 1773). EM: Cascada Los Diamantes, San Rafael XII (MZFC), (Barrera \& Romero 1986). 
Cyanophrys goodsoni (Clench 1946). EM: Sierra Nevada (FES-Z: Luna-Reyes \& Llorente-Bousquets 2004).

Cyanophrys herodotus (Fabricius 1793). MALI: El Molino IV (CNIN: HernándezMejía 2005).

Cyanophrys longula (Hewitson 1868). CHIA: Tenancingo X (CNIN); EM: Sierra Nevada (FES-Z: Luna-Reyes \& Llorente-Bousquets 2004); MALI: Malinalco X (CNIN); OA: Ocuilan-Cuernavaca, km 14 I,III (MZFC); TEM: El Polvorín I (MZFC); VG: Villa Guerrero V (MZFC); ST: Santo Tomás de los Plátanos (De la Maza 1987).

Callophrys spinetorum (Hewitson 1867). EM: Sierra Nevada (FES-Z: Luna-Reyes \& Llorente-Bousquets 2005).

Callophrys xami (Reakirt [1867]). OA: Ocuilan-Cuernavaca, km 14 I (MZFC).

*Laothus erybathis (Hewitson 1867). EM: carretera Villa Victoria-Valle de Bravo, km 22 XI (CNIN); OA: Ocuilan-Cuernavaca, km 14 I (MZFC); TEM: El Polvorín I (MZFC); VG: Villa Guerrero IX,XI (CNIN); ZAC: Zacualpan II (MZFC), Tres Cruces de Mamatla XII (MZFC).

Lamprospilus sethon (Godman \& Salvin 1887). NAU: Naucalpan (CNIN); VG: Villa Guerrero V (MZFC).

Ziegleria syllis (Godman \& Salvin 1887). MALI: Camino viejo a Ocuilan VII (CNIN: Hernández-Mejía 2005).

Ziegleria guzanta (Schaus 1902). MALI: Camino viejo a Ocuilan VII (CNIN: Hernández-Mejía 2005); OA: Ocuilan XII (MZFC); VG: Villa Guerrero IV (CNIN); ZAC: Tres Cruces de Mamatla XII (MZFC).

Electrostrymon mathewi (Hewitson 1874). MALI: El Molino IV (CNIN: HernándezMejía 2005).

Electrostrymon sangala (Hewitson 1868). EM: San Nicolás Tolentino (De la Maza 1987).

Calycopis clarina (Hewitson 1874). MALI: El Molino IV (CNIN: Hernández-Mejía 2005).

Calycopis isobeon (Butler \& H. Druce 1872). MALI: Colonia Juárez III, El Molino IV, Puente Caporal IX (CNIN: Hernández-Mejía 2005).

Strymon melinus (Hübner [1813]). CHIA: Tenancingo X (CNIN); EM: Salto de Santana VII (CNIN).

Strymon yojoa (Reakirt [1867]). MALI: La Angostura XI, Puente Caporal X (CNIN: Hernández-Mejía 2005); OA: Ocuilan-Cuernavaca, km 14 (MZFC).

Strymon cestri (Reakirt [1867]). MALI: Jalmolonga V (CNIN: Hernández-Mejía 2005).

Strymon astiocha (Prittwitz 1865). MALI: Puente Caporal IX,XII (CNIN: HernándezMejía 2005).

Strymon bazochii (Godart [1824]). ZAC: Puerto Obscuro II (MZFC).

Strymon istapa (Reakirt [1867]). MALI: Jalmolonga V, La Angostura XI, Puente Caporal XII (CNIN: Hernández-Mejía 2005); OA: Ocuilan-Cuernavaca, km 14 I (MZFC). 
Strymon serapio (Godman \& Salvin 1887). MALI: El Molino IX (CNIN: Hernández-Mejía 2005).

Strymon ziba (Hewitson 1868). NAU: Naucalpan V (CNIN).

Tmolus echion (Linnaeus 1767). MALI: El Molino IV (CNIN: Hernández-Mejía 2005).

Ministrymon clytie (W.H. Edwards 1877). MALI: Tepehuajes V (CNIN: HernándezMejía 2005).

Ministrymon azia (Hewitson 1873). CUA: Cuautitlán VII (CNIN); MALI: San Andrés Nicolás Bravo XI, El Molino IV (CNIN: Hernández-Mejía 2005).

Theclopsis mycon (Godman \& Salvin 1887). AZ: Atizapán de Zaragoza (USNM).

Panthiades bitias (Cramer 1777). MALI: El Molino IV (CNIN: Hernández-Mejía 2005).

Panthiades bathildis (C. Felder \& R. Felder 1865). CHIA: Tenancingo X (CNIN); MALI: El Molino V (CNIN: Hernández-Mejía 2005).

Parrhasius moctezuma (Clench 1971). OA: Ocuilan-Cuernavaca, km 14 I (MZFC); TEM: El Polvorín I (MZFC).

Michaelus jebus (Godart [1824]). EM: San Nicolás XII (CNIN).

Aubergina paetus (Godman \& Salvin 1887). MALI: El Molino IX, Colonia Juárez IV (CNIN: Hernández-Mejía 2005).

Erora quaderna (Hewitson 1868). EM: Cascada Los Diamantes, San Rafael (Barrera \& Romero 1986); Sierra Nevada (FES-Z: Luna-Reyes \& Llorente-Bousquets 2004); OA: Ocuilan-Cuernavaca, km 14 I (MZFC); TEM: El Polvorín I (MZFC); ZAC: Puerto Obscuro VIII (MZFC).

Erora subflorens (Schaus 1913). ORO: El Oro IX (MZFC).

Chalybs hassan (Stoll 1790). MALI: El Molino IV, San Andrés Nicolás Bravo XI (CNIN: Hernández-Mejía 2005).

SUBFAMILIA POLYOMMATINAE

Leptotes cassius cassidula (Boisduval 1870). MALI: Colonia Juárez VI, El Molino IV-V, El Platanar VII,IX (CNIN: Hernández-Mejía 2005).

Leptotes marina (Reakirt 1868). CUA: Cuautitlán VII (CNIN); EM: Sierra Nevada (FES-Z: Luna-Reyes \& Llorente-Bousquets 2004); MALI: Chalma (Guzmán 1976); Camino viejo a Ocuilan VII, Cerro "El Zapote" VII, El Molino IV-V, Jalmolonga V, Tepehuajes VIII (CNIN: Hernández-Mejía 2005); TEM: Parque Universitario "Las Orquídeas" IV,XII (Aguilera-Ríos y Hernández-Mejía, en prep.); TEO: Teotihuacán VI (USNM); ZAC: Puerto Obscuro XI (MZFC); TEJ: Cañada La Canoa, 2.5 km del Reparo (Barrera \& Díaz-Batres 1977).

Zizula cyna (W.H. Edwards 1881). MALI: El Platanar I (CNIN: Hernández-Mejía 2005).

Cupido comyntas (Godart [1824]). ALQ: Almoloya de Alquisiras III (CNIN); MALI: El Molino V,IX, San Pedro Chichicasco IX (CNIN: Hernández-Mejía 2005).

Celastrina argiolus gozora (Boisduval 1870). AZ: Atizapán de Zaragoza VII (MZFC); EM: Cascada Los Diamantes, San Rafael (Barrera \& Romero 1986); 
El Polvorín I (MZFC); Lagunas de Zempoala IV (CNIN); Sierra Nevada (FESZ: Luna-Reyes \& Llorente-Bousquets 2004); MALI: Puente Caporal X (CNIN: Hernández-Mejía 2005); TEJ: Cañada La Canoa, 2.5 km del Reparo (Barrera \& Díaz-Batres 1977); TEM: Parque Universitario "Las Orquídeas” XII (AguileraRíos y Hernández-Mejía, en prep.).

Hemiargus hanno antibubastus Hübner [1818]. ALQ: Almoloya de Alquisiras IX (CMNH); EM: (AMNH); Sierra Nevada (FES-Z: Luna-Reyes \& LlorenteBousquets 2004); MALI: Colonia Juárez IV,VI, El Molino IV, El Platanar VII, Jalmolonga V, Puente Caporal X, San Andrés Nicolás Bravo VI, San Pedro Chichicasco V, Tepehuajes V (CNIN: Hernández-Mejía 2005); TEJ: Cañada La Canoa, 2.5 km del Reparo y El Estanco, Sierra de Nanchititla (Barrera \& DíazBatres 1977).

Echinargus isola (Reakirt [1867]). EM: (AMNH), carretera Tlalóc X (CNIN); Cascada Los Diamantes, San Rafael (Barrera \& Romero 1986); El Polvorín I (MZFC); Popocatépetl VI,XII (USNM); San Bartolo XII (CNIN); Sierra Nevada (FES-Z: Luna-Reyes \& Llorente-Bousquets 2004); MALI: Chalma (Guzmán 1976); El Molino IV,IX, El Platanar XI, Puente Caporal IX,XII, Tepehuajes V (CNIN: Hernández-Mejía 2005); JIL: Jilotepec X (CNIN); TLAL: Tlalnepantla (USNM); TOL: Toluca XI (CNIN); El Cerrillo Piedras Blancas IX-XI; ZAC: Puerto Obscuro II,XI (MZFC).

Aricia acmon (Westwood [1851]). AMEC: Amecameca XI (CNIN); EM: Cascada Los Diamantes (Barrera \& Romero 1986); Sierra Nevada (FES-Z: Luna-Reyes \& Llorente-Bousquets 2004); Vista Hermosa (carretera a Toluca) VIII (CNIN); ST: Santo Tomás de los Plátanos IX (CNIN).

FAMILIA RIODINIDAE

SUBFAMILIA EUSELASIINAE

Euselasia aurantiaca aurantiaca (Salvin \& Godman 1868). TEJ: Cañada Bosque Encantado (Barrera \& Díaz-Batres 1977).

\section{SUBFAMILIA RIODININAE}

Napaea umbra (Boisduval 1870). MALI: Malinalco VIII (MCLB).

*Rhetus arcius beutelspacheri Llorente 1988. EM: San Nicolás Tolentino (De la Maza 1987); MALI: Colonia Juárez III (CNIN: Hernández-Mejía 2005).

Calephelis perditalis perditalis Barnes \& McDunnough 1918. EM: Sierra Nevada (FES-Z: Luna-Reyes \& Llorente-Bousquets 2004).

Calephelis sixola McAlpine 1971. MALI: Colonia Juárez IV, La Angostura XI, Puente Caporal X (CNIN: Hernández-Mejía 2005).

Calephelis sp. MALI: Colonia Juárez IV, El Molino IX, La Angostura VI,XI, Puente Caporal X, San Andrés Nicolás Bravo II,VI (CNIN: Hernández-Mejía 2005).

Baeotis zonata zonata R. Felder 1869. EM: San Nicolás Tolentino (De la Maza 1987); MALI: Malinalco VIII (AME); Puente Caporal XII (CNIN: HernándezMejía 2005); NR: Nicolás Romero III,V (CNIN).

Lasaia maria maria Clench 1972. MALI: Malinalco VII (MCLB), VIII (MZFC), VII (AME); El Platanar VII (CNIN: Hernández-Mejía 2005). 
Melanis pixe pixe (Boisduval 1836). ST: Santo Tomás de los Plátanos V (CNIN).

*Melanis cephise cephise (Ménétriés 1855). MALI: San Pedro Chichicasco IX (CNIN: Hernández-Mejía 2005); ST: Santo Tomás de los Plátanos V (CNIN).

Anteros carausius carausius Westwood 1851. EM: San Nicolás Tolentino (De la Maza 1976); ST: Santo Tomás de los Plátanos (De la Maza 1976).

Emesis saturata Godman \& Salvin 1886. MALI: El Platanar IX, La Angostura XI, Puente Caporal X, San Pedro Chichicasco V (CNIN: Hernández-Mejía 2005); ZAC: Puerto Obscuro I (MZFC).

Emesis mandana furor Butler \& H. Druce 1872. EM: San Nicolás Tolentino (De la Maza 1978); Sierra Nevada (FES-Z: Luna-Reyes \& Llorente-Bousquets 2004).

Emesis vulpina Godman \& Salvin 1886. MALI: Camino viejo a Ocuilan VII, Colonia Juárez X, San Andrés Nicolás Bravo II (CNIN: Hernández-Mejía 2005).

Emesis tenedia C. Felder \& R. Felder 1861. EM: Carretera Villa Victoria-Valle de Bravo, km 22 IV (CNIN); MALI: Malinalco VIII (MCLB), VIII (AME); Colonia Juárez IV, El Platanar XI, La Angostura II, San Andrés Nicolás Bravo XI (CNIN: Hernández-Mejía 2005); ST: Santo Tomás de los Plátanos III (CNIN).

Emesis lupina lupina Godman \& Salvin 1886. MALI: Malinalco VIII (MCLB), VIII (AME).

*Emesis zela Butler 1870. ALQ: Almoloya de Alquisiras VIII (AME); AMEC: Amecameca (Godman \& Salvin 1878-1901); ATZ: Atizapán VI,VIII (MCLB); AZ: Atizapán de Zaragoza VI,VIII (AME); CHIA: Tenancingo VIII (MZFC), III,IX (AME); EM: Almoloya IX (MCLB); Almoloya VIII (MCLB); Avándaro VI (AME), VI (MCLB); Cascada los Diamantes, San Rafael (Barrera \& Romero 1986); El Polvorín I (MZFC); Popocatépetl (USNM); Sierra Nevada (FESZ: Luna-Reyes \& Llorente-Bousquets 2004); IXS: Grutas de La Estrella VII (CNIN); MALI: Malinalco XII (MCLB), XII (AME); Cerro "El Zapote" VII (CNIN: Hernández-Mejía 2005); TEN: Tenancingo IX (MCLB); TOL: Toluca XI (CNIN); ZAC: Puerto Obscuro II (MZFC).

Apodemia walkeri Godman \& Salvin 1886. MALI: El Platanar XI, San Andrés Nicolás Bravo XI (CNIN: Hernández-Mejía 2005).

Synargis mycone (Hewitson 1865). MALI: San Andrés Nicolás Bravo IV (CNIN: Hernández-Mejía 2005).

*Theope villai Beutelspacher 1981. EM: San Nicolás Tolentino (De la Maza 1987). FAMILIA NYMPHALIDAE

SUBFAMILIA LIBYTHEINAE

Libytheana carinenta mexicana Michener 1943. EM: Sierra Nevada (FES-Z: LunaReyes \& Llorente-Bousquets 2004); MALI: Colonia Juárez VI, El Platanar XI, La Angostura VI (CNIN: Hernández-Mejía 2005).

SUBFAMILIA DANAINAE

Anetia thirza thirza Geyer [1833]. ATL: Atlauta IV (CNIN); AMEC: Amecameca (USNM); EM: Cascada Los Diamantes, San Rafael II,IV-V (MZFC), (Barrera \& Romero 1986); Popocatépetl (De la Maza 1987); Sierra Nevada (FES-Z: LunaReyes \& Llorente-Bousquets 2004). 
Lycorea halia atergatis Doubleday [1847]. TEX: Chapingo (COEE: Gibson \& Carrillo 1959).

Danaus eresimus montezuma Talbot 1943. MALI: Puente Caporal XII, San Andrés Nicolás Bravo IV (CNIN: Hernández-Mejía 2005).

Danaus gilippus thersippus (H. W. Bates 1863). ATZ: Ciudad A. López Mateos VIII (CNIN); EM: Cascada Los Diamantes, San Rafael I (MZFC), (Barrera \& Romero 1976); Popocatépetl (AMNH); San Nicolás VI (CNIN), (USNM); Sierra Nevada (Luna-Reyes \& Llorente-Bousquets 2004); IXS: Grutas de La Estrella VII (CNIN); MALI: Chalma (Guzmán 1976); Colonia Juárez III-IV, El Molino IV-V, Jalmolonga V, La Angostura X, Puente Caporal XII, San Pedro Chichicasco V (CNIN: Hernández-Mejía 2005); NR: Nicolás Romero I (CNIN); TEJ: El Estanco, Sierra de Nanchititla (Barrera \& Díaz-Batres 1977); TON: Camino al Cerro de la Cruz XII (CNIN); VB: Colorines (De la Maza 1987); ZUM: Zumpango VIII (CNIN).

Danaus plexippus plexippus (Linnaeus 1758). ATZ: Ciudad A. López Mateos VIII (CNIN); DG: Donato Guerra (De la Maza 1987); EM: Cascada Los Diamantes, San Rafael II,IX,XI-XII (MZFC), (Barrera \& Romero 1986); Cerro Pelón (De la Maza 1987); Cerro Piedra Herrada, cerca de Valle de Bravo (Calvert \& Brower 1986); Popocatépetl VIII (MZFC); San Nicolás I (CNIN); Sierra Nevada (FESZ: Luna-Reyes \& Llorente-Bousquets 2004); MALI: Jalmolonga V (CNIN: Hernández-Mejía 2005); OA: Ocuilan-Cuernavaca, km 14 III (MZFC); TOL: El Cerrillo Piedras Blancas VI; TON: Camino al Cerro de la Cruz XII (CNIN); VB: Colorines (De la Maza 1987).

SUBFAMILIA MORPHINAE

Morpho polyphemus polyphemus Westwood [1850]. EM: Ixtapantongo (De la Maza 1987); San Nicolás IX (CNIN); Sierra Nevada (FES-Z: Luna-Reyes \& LlorenteBousquets 2004); MALI: Malinalco VIII,X (AME), V (CAS); Chalma (Guzmán 1976), (CNIN); Camino viejo a Ocuilan VII, El Molino V, El Platanar V (CNIN: Hernández-Mejía 2005); ST: Santo Tomás de los Plátanos V (CNIN), (De la Maza 1987); TEJ: Cañada La Canoa, 2.5 km del Reparo y El Estanco, Sierra de Nanchititla (Barrera \& Díaz-Batres 1977); TEM: Parque Universitario "Las Orquídeas” XII (Aguilera-Ríos y Hernández-Mejía, en prep.).

Opsiphanes boisduvallii Doubleday [1849]. VB: Colorines (De la Maza 1987).

SUBFAMILIA SATYRINAE

Manataria hercyna maculata (Hopffer 1874). MALI: Colonia Juárez VI (CNIN: Hernández-Mejía 2005); TEJ: Nanchititla (De la Maza 1987); Cañada Bosque Encantado (Barrera \& Díaz-Batres 1977).

Oxeoschistus tauropolis ssp. nov. VB: Valle de Bravo X (AME).

Cissia similis (Butler 1867). MALI: Colonia Juárez VI (CNIN: Hernández-Mejía 2005); ST: Santo Tomás de los Plátanos III (CNIN).

Cissia themis (Butler 1867). CUA: Cuautitlán I (MZFC); MALI: Tepehuajes VIII, Colonia Juárez V (CNIN: Hernández-Mejía 2005).

*Cyllopsis diazi L.D. Miller 1974. MALI: Malinalco (AME: Miller 1974); OA: Ocuilan-Cuernavaca, km 14 I (MZFC). 
*Cyllopsis henshawi hoffmanni L.D. Miller 1974. AZ: Atizapán de Zaragoza VI (CNIN); MALI: Malinalco VI (MZFC); TEN: Tenancingo (AME: Miller 1974); VG: Villa Guerrero X-XI (CNIN).

Cyllopsis hilaria (Godman 1901). MALI: San Pedro Chichicasco II (CNIN: Hernández-Mejía 2005); ZAC: Puerto Obscuro II (MZFC).

*Cyllopsis nayarit R.L. Chermock 1947. MALI: Malinalco (AME: Miller 1974).

*Cyllopsis parvimaculata L.D. Miller 1974. TEN: Tenancingo (AME), (Miller 1974), (Miller \& De la Maza 1984).

Cyllopsis pephredo (Godman 1901). MALI: Puente Caporal X (CNIN: HernándezMejía 2005); OA: Ocuilan-Cuernavaca, km 14 I (MZFC); VG: Villa Guerrero XI (CNIN).

*Cyllopsis perplexa L.D. Miller 1974. EM: Cerro San Miguel IV (MZFC); MALI: Malinalco XI (MZFC); OA: Ocuilan-Cuernavaca, km 14 I,III (MZFC); TEN: Tenancingo (AME: Miller 1974); ZAC: Puerto Obscuro XI (MZFC); Tres Cruces de Mamatla XII (MZFC).

*Cyllopsis pertepida pertepida (Dyar 1912). ATZ: Atizapán (Miller 1974); AZ: Atizapán de Zaragoza VI-VII (CNIN); JIL: Jilotepec X (CNIN).

Cyllopsis pyracmon pyracmon (Butler 1867). EM: Sierra Nevada (FES-Z: LunaReyes \& Llorente-Bousquets 2004); OA: Ocuilan-Cuernavaca, km 14 I,III (MZFC); VG: Villa Guerrero I,VI,X (CNIN); ZAC: Tres Cruces de Mamatla XII (MZFC).

Cyllopsis steinhauserorum L.D. Miller 1974. OA: Ocuilan-Cuernavaca, km 14 I (MZFC).

*Cyllopsis windi L.D. Miller, 1974. OA: Ocuilan-Cuernavaca, km 14 I (MZFC).

*Euptychia fetna Butler 1870. MALI: Cerro "El Zapote" VII, La Angostura VI (CNIN: Hernández-Mejía 2005).

Hermeuptychia hermes (Fabricius 1775). MALI: El Molino IV,IX, El Platanar I, La Angostura VI, Puente Caporal IX-X, Tepehuajes VIII (CNIN: Hernández-Mejía 2005).

Megisto rubricata anabelae L.D. Miller 1976. AZ: Atizapán de Zaragoza VI (CNIN), (De la Maza 1987); EM: San Mateo Nopala (De la Maza 1987); MALI: Chalma (Guzmán 1976).

*Paramacera xicaque xicaque (Reakirt [1867]). AMEC: Amecameca (Godman \& Salvin 1878-1901); EM: Cascada Los Diamantes, San Rafael (Barrera \& Romero 1986); Lagunas de Zempoala XII (CNIN); Llano Grande IV,XI (CNIN); Popocatépetl (De la Maza 1987), VI (USNM); Río Frío VI (MZFC); San Cayetano X (CNIN); Sierra Nevada (FES-Z: Luna-Reyes \& Llorente-Bousquets 2004); TOL: $64 \mathrm{~km}$ al W de Toluca VI.

Pareuptychia ocirrhoe ssp. n. TEX: Chapingo (INIA: Domínguez \& Carrillo 1976).

Pindis squamistriga R. Felder 1869. EM: Sierra Nevada (FES-Z: Luna-Reyes \& Llorente-Bousquets 2004); MALI: Chalma (Guzmán 1976); Malinalco (AME: Miller 1978); Tepehuajes VIII (CNIN: Hernández-Mejía 2005); OA: OcuilanCuernavaca km 14 I (MZFC); TEJ: Cañada Bosque Encantado (Barrera \& DíazBatres 1977); TEN: Tenancingo (AME: Miller 1978). 
*Taygetis mermeria griseomarginata L.D. Miller 1978. TEJ: Cañada Bosque Encantado (Barrera \& Díaz-Batres 1977).

*Taygetis weymeri Draudt 1912. MALI: Colonia Juárez VI (CNIN: Hernández-Mejía 2005); TEJ: Cañada Bosque Encantado (Barrera \& Díaz-Batres 1977).

Yphthimoides renata (Stoll 1780). MALI: Malinalco VIII (AME).

Zischkaia lupita (Reakirt [1867]). MALI: Chalma (Guzmán 1976).

Gyrocheilus patrobas patrobas (Hewitson 1862). CHIA: Tenancingo VIII (MZFC); NAU: Naucalpan X (CNIN).

\section{SUBFAMILIA CHARAXINAE}

Anaea troglodyta aidea (Guérin-Méneville [1844]). EM: Cascada los Diamantes, San Rafael II,V (MZFC: Barrera \& Romero 1986); MALI: Chalma (Guzmán 1976); El Platanar VII,XI, La Angostura VI, San Andrés Nicolás Bravo VIII, Tepehuajes VIII (CNIN: Hernández-Mejía 2005).

Memphis hedemanni (R. Felder 1869). MALI: Malinalco (MCLB).

*Archaeoprepona demophon occidentalis Stoffel \& Descimon 1974. MALI: Colonia Juárez III, San Andrés Nicolás Bravo XII (CNIN: Hernández-Mejía 2005).

\section{SUBFAMILIA BIBLIDINAE}

Marpesia chiron marius (Cramer 1779). AZ: Atizapán de Zaragoza VII (MZFC).

Marpesia petreus ssp. n. MALI: El Platanar XI (CNIN: Hernández-Mejía 2005).

Biblis hyperia aganisa Boisduval 1836. MALI: Colonia Juárez IV (CNIN: HernándezMejía 2005); VB: Colorines (De la Maza 1987).

Mestra dorcas amymone (Ménétriés 1857). EM: Zirimicuaro XII (MZFC); MALI: Colonia Juárez X (CNIN: Hernández-Mejía 2005); ST: Santo Tomás de los Plátanos V (CNIN).

Myscelia cyananthe cyananthe C. Felder \& R. Felder 1867. MALI: Chalma (Guzmán 1976); El Platanar XI, La Angostura X, Puente Caporal X, San Andrés Nicolás Bravo II,IV (CNIN: Hernández-Mejía 2005).

Myscelia ethusa ethusa (Doyère [1840]). TOL: Toluca IX (MZFC).

*Hamadryas atlantis lelaps Godman \& Salvin 1883. EM: Ixtapantongo (De la Maza 1987); MALI: Malinalco (Jenkins 1983); TEJ: Nanchititla (De la Maza 1987).

*Hamadryas guatemalena marmarice (Fruhstorfer 1916). AZ: Atizapán de Zaragoza IV (MZFC); TEJ: Cañada Bosque Encantado (Barrera \& Díaz-Batres 1977).

*Bolboneura sylphis beatrix R.G. Maza 1985. MALI: Colonia Juárez X, La Angostura X, San Andrés Nicolás Bravo VI (CNIN: Hernández-Mejía 2005).

*Epiphile adrasta escalantei Descimon \& Mast 1979. EM: carretera México-Toluca, km 18 X (CNIN), (De la Maza \& Turrent 1986); San Nicolás (CDM), (Descimon \& Mast de M. 1979), (Jenkins 1986); MALI: Chalma y Malinalco (AME: Jenkins 1986); Puente Caporal X (CNIN: Hernández-Mejía 2005); OA: OcuilanCuernavaca, km 14 III (MZFC); TEM: Parque Universitario “Las Orquídeas” XI (Aguilera-Ríos y Hernández-Mejía, en prep.).

*Temenis laothoe quilapayunia R.G. Maza \& Turrent 1985. MALI: El Platanar V (CNIN: Hernández-Mejía 2005). 
Diaethria anna mixteca J. Maza 1997. TEM: Parque Universitario "Las Orquídeas" IX (Aguilera-Ríos y Hernández-Mejía, en prep.).

Ciclogramma bacchis (Doubleday 1849). EM: Sierra Nevada (FES-Z: Luna-Reyes \& Llorente-Bousquets 2004); MALI: El Platanar VII, Puente Caporal X (CNIN: Hernández-Mejía 2005); OA: Ocuilan-Cuernavaca, km 14 I,III (MZFC); VB: Colorines (De la Maza 1987), (De la Maza \& Turrent 1985); VG: Villa Guerrero (De la Maza \& Turrent 1985).

Adelpha bredowii bredowii Geyer 1837. AMEC: Amecameca (Godman \& Salvin 1878-1901); AZ: Atizapán de Zaragoza VII (CNIN); CHIA: Tenancingo VIII (MZFC); EM: carretera Villa Victoria-Valle de Bravo, km 22 IV (CNIN); MALI: Chalma VII (AME); OA: Ocuilan-Cuernavaca, km 14 III (MZFC); VB Valle de Bravo (De la Maza 1987).

Adelpha donysa donysa (Hewitson 1847). VB: Valle de Bravo XI (AME).

Adelpha fessonia fessonia (Hewitson 1847). EM: Nuevo Morelos XI (AME); NR: Nicolás Romero V (CNIN).

Adelpha paraena massilia (C. Felder \& R. Felder 1867). MALI: VIII,XI (AME); Chalma VII (AME); VB: Valle de Bravo XI (AME).

Adelpha serpa celerio (H.W. Bates 1864). EM: carretera Villa Victoria-Valle de Bravo, Méx. Km 22 (CNIN).

SUBFAMILIA APATURINAE

Asterocampa idyja argus (H.W. Bates, 1864). EM: Zirimicuaro XI (MZFC); MALI: La Angostura X (CNIN: Hernández-Mejía 2005); OA: Ocuilan-Cuernavaca, km 14 III (MZFC).

Doxocopa laure laure (Drury 1773). EM: San Nicolás V (CNIN); Sierra Nevada (FES-Z: Luna-Reyes \& Llorente-Bousquets 2004); MALI: La Angostura X, Puente Caporal IX, Colonia Juárez III (CNIN: Hernández-Mejía 2005); OA: Ocuilan-Cuernavaca, km 14 III (MZFC); ST: Santo Tomás de los Plátanos (De la Maza 1987).

\section{SUBFAMILIA NYMPHALINAE}

Smyrna karwinskii Geyer [1833]. EM: San Nicolás IX (CNIN); MALI: San Andrés Nicolás Bravo VI (CNIN: Hernández-Mejía 2005).

Nymphalis antiopa antiopa (Linnaeus 1758). EM: Cascada Los Diamantes, San Rafael I-III (MZFC), (Barrera \& Romero 1986); Popocatépetl VI (USNM); Sierra Nevada (FES-Z: Luna-Reyes \& Llorente-Bousquets 2004); MALI: Puente Caporal X (CNIN: Hernández-Mejía 2005); TEX: Chapingo VIII (MZFC), (COEE: Gibson \& Carrillo 1959); TOL: El Cerrillo Piedras Blancas VI.

*Polygonia haroldii (Dewitz 1877). AMEC: Amecameca (Godman \& Salvin 18781901); EM: Cascada Los Diamantes I-III,V-VI,X-XII (MZFC), (Barrera \& Romero 1986); Popocatépetl III (MZFC) (Hoffmann 1940), VI (USNM); Sierra Nevada (FES-Z: Luna-Reyes \& Llorente-Bousquets 2004).

Polygonia sp. EM: Sierra Nevada (FES-Z: Luna-Reyes \& Llorente-Bousquets 2004).

Vanessa annabella (Field 1971). AZ: Atizapán de Zaragoza VIII (CNIN); CHIA: Tenancingo VII (MZFC); EM: Cascada Los Diamantes, San Rafael II,X-XI 
(MZFC), (Barrera \& Romero 1986); Sierra Nevada (FES-Z: Luna-Reyes \& Llorente-Bousquets 2004); TEX: Chapingo (COEE: Gibson \& Carrillo 1959); VB: Valle de Bravo (De la Maza 1987).

Vanessa atalanta rubria (Fruhstorfer 1909). EM: Cerro San Miguel XI (MZFC); Popocatépetl VI (USNM); Sierra Nevada (FES-Z: Luna-Reyes \& LlorenteBousquets 2004); TEX: Texcoco IX (MZFC); VB: Valle de Bravo (De la Maza 1987).

Vanessa cardui (Linnaeus 1758). AZ: Atizapán de Zaragoza VI (MZFC); EM: Sierra Nevada (FES-Z: Luna-Reyes \& Llorente-Bousquets 2004); MALI: El Platanar VII, San Andrés Nicolás Bravo XI (CNIN: Hernández-Mejía 2005); TEX: Chapingo X (MZFC).

Vanessa virginiensis (Drury 1773). AZ: Atizapán de Zaragoza VIII (CNIN); EM: Cascada Los Diamantes, San Rafael I-II,IV (MZFC), (Barrera \& Romero 1986); Sierra Nevada (FES-Z: Luna-Reyes \& Llorente-Bousquets 2004); MALI: San Pedro Chichicasco V (CNIN: Hernández-Mejía 2005).

Anartia fatima fatima (Fabricius 1793). MALI: Colonia Juárez V-VI, El Platanar V, San Andrés Nicolás Bravo VI (CNIN: Hernández-Mejía 2005); TEJ: Cañada la Canoa, $2.5 \mathrm{~km}$ del Reparo y El Estanco, Sierra de Nanchititla (Barrera \& DíazBatres 1977).

Anartia jatrophae luteipicta Fruhstorfer 1907. MALI: Puente Caporal XI, Tepehuajes VIII (CNIN: Hernández-Mejía 2005); TEJ: El Estanco, Sierra de Nanchititla (Barrera \& Díaz-Batres 1977).

Junonia coenia Hübner [1822]. EM: Cascada Los Diamantes, San Rafael II,IV (MZFC), (Barrera \& Romero 1986); Sierra Nevada (FES-Z: Luna-Reyes \& Llorente-Bousquets 2004); MALI: Colonia Juárez VI, La Angostura XI, Puente Caporal X (CNIN: Hernández-Mejía 2005); OA: Ocuilan-Cuernavaca, km 14 I,III (MZFC); ST: Santo Tomás de los Plátanos (De la Maza 1987); TEJ: Tejupilco, 10 km W VII (MZFC); VB: Colorines (De la Maza 1987); ZAC: Puerto Obscuro II (MZFC); Tres Cruces de Mamatla XII (MZFC).

Junonia evarete ssp. n. MALI: Chalma (Guzmán 1976).

Siproeta stelenes biplagiata (Fruhstorfer 1907). ALQ: Almoloya de Alquisiras V (MZFC); EM: Cascada Los Diamantes (Barrera \& Romero 1986); Sierra Nevada (FES-Z: Luna-Reyes \& Llorente-Bousquets 2004); MALI: Chalma (Guzmán 1976); San Andrés Nicolás Bravo II (CNIN: Hernández-Mejía 2005); OA: Ocuilan-Cuernavaca, km 14 III (MZFC); ST: Santo Tomás de los Plátanos V (CNIN); TEJ: Cañada La Canoa, 2.5 km del Reparo (Barrera \& Díaz-Batres 1977).

Chlosyne cyneas cyneas (Godman \& Salvin 1878). EM: Popocatépetl VII (USNM), (MCLB); Sierra Nevada (FES-Z: Luna-Reyes \& Llorente-Bousquets 2004).

Chlosyne definita definita (Aaron [1885]). ATZ: Atizapán (MCLB); AZ: Atizapán de Zaragoza VII-VIII (CNIN); JIL: Jilotepec X (CNIN).

*Chlosyne ehrenbergii (Geyer, [1833]). ALQ: Almoloya de Alquisiras V (MZFC); AMEC: Amecameca IX (CMNH), (Godman \& Salvin 1878-1901); ATZ: Atizapán (MCLB); CHIA: Tenancingo VIII (MZFC); EM: Popocatépetl VII (USNM); Sierra Nevada (FES-Z: Luna-Reyes \& Llorente-Bousquets 2004); 
MALI: Malinalco (MCLB); Chalma (Guzmán 1976), (Kendall \& McGuire 1984); Colonia Juárez IV, Jalmolonga V, San Pedro Chichicasco V (CNIN: Hernández-Mejía 2005); OA: Ocuilan-Cuernavaca, km 14 III (MZFC); TEJ: El Estanco, Sierra de Nanchititla (Barrera \& Díaz-Batres 1977); Nanchititla (Kendall \& McGuire 1984); TEN: Tenancingo (MCLB); TEO: Ruinas de Teotihuacán (MCLB); TEX: El Molino de las Flores (INIA: Domínguez \& Carrillo 1976); TUL: Tultepec VII (MZFC); VB Valle de Bravo VIII (UCB).

Chlosyne hippodrome hippodrome (Geyer 1837). EM: Ixtapantongo X (CNIN); MALI: El Platanar VIII, San Pedro Chichicasco IX (CNIN: Hernández-Mejía, 2005); TEM: Parque Universitario "Las Orquídeas" V,XI (Aguilera-Ríos y Hernández-Mejía, en prep.).

Chlosyne janais janais (Drury 1782). MALI: Colonia Juárez III (CNIN: HernándezMejía 2005).

Chlosyne lacinia crocale (W.H. Edwards 1874). ATZ: Atizapán (MCLB); AZ: Atizapán de Zaragoza VI (MZFC), I,VIII (CNIN); EM: (AMNH); Popocatépetl (Higgins 1960); Sierra Nevada (FES-Z: Luna-Reyes \& Llorente-Bousquets 2004); IXS: Ixtapan de la Sal (MCLB); MALI: Chalma (Guzmán 1976); El Molino V, El Platanar IX, San Andrés Nicolás Bravo VI, Tepehuajes VIII (CNIN: Hernández-Mejía 2005); TEJ: El Estanco, Sierra de Nanchititla (Barrera \& DíazBatres 1977).

*Chlosyne marina eumeda (Godman \& Salvin 1894). CHIA: Tenancingo VIII (MZFC); CUA: Cuautitlán VIII (MZFC); MALI: Malinalco (MCLB); Chalma (Guzmán 1976).

*Chlosyne rosita riobalsensis Bauer 1961. MALI: El Platanar VII, Puente Caporal X, San Andrés Nicolás Bravo X (CNIN: Hernández-Mejía 2005).

Chlosyne theona theona (Ménétriés 1855). ATZ: Ciudad A. López Mateos VIII (CNIN); AZ: Atizapán de Zaragoza VII (MZFC); EM: Llanos del Peñón IV (AMNH); Los Remedios VIII; Los Reyes, 2 mi SE VII (UCB); IXS: Ixtapan de la Sal VII.

Microtia elva elva H.W. Bates 1864. EM: (AMNH); MALI: El Platanar V,VII, La Angostura VI, San Andrés Nicolás Bravo VI,VIII, San Pedro Chichicasco V (CNIN: Hernández-Mejía 2005).

*Texola anomalus coracara (Dyar 1912). MALI: San Pedro Chichicasco IX (CNIN: Hernández-Mejía 2005).

Texola elada elada (Hewitson 1868). AZ: Atizapán de Zaragoza VII (MZFC); EM: Hacienda Caracha VI (MZFC); Sierra Nevada (FES-Z: Luna-Reyes \& LlorenteBousquets 2004); MALI: Cerro "El Zapote" VII, La Angostura VI,X, San Andrés Nicolás Bravo VI,VIII, Tepehuajes V (CNIN: Hernández-Mejía 2005).

Anthanassa ardys ardys (Hewitson 1864). EM: El Polvorín I (MZFC); MALI: Colonia Juárez III, El Molino IV, Puente Caporal X, Tepehuajes V (CNIN: Hernández-Mejía 2005).

Anthanassa frisia tulcis (H.W. Bates 1864). MALI: San Andrés Nicolás Bravo II (CNIN: Hernández-Mejía 2005).

*Anthanassa nebulosa alexon (Godman \& Salvin 1889). EM: El Polvorín I (MZFC); San Nicolás III (CNIN); Sierra Nevada (FES-Z: Luna-Reyes \& 
Llorente-Bousquets 2004); MALI: Cerro “El Zapote” VII, Colonia Juárez III, El Molino V, El Platanar VII, La Angostura II, Puente Caporal II,IX, San Andrés Nicolás Bravo VI, (CNIN: Hernández-Mejía 2005); OA: Ocuilan-Cuernavaca, km 14 I,III (MZFC).

*Anthanassa ptolyca amator (A. Hall 1929). EM: San Nicolás III (CNIN); OA: Ocuilan-Cuernavaca, km 14 I,III,VI (MZFC); ZAC Tres Cruces de Mamatla II,XI (MZFC).

*Anthanassa sitalces cortes (A. Hall 1917). AMEC: Amecameca X (MZFC).

Anthanassa texana texana (W.H. Edwards 1863). ALQ: Almoloya de Alquisiras V (MZFC); AZ: Atizapán de Zaragoza VI (MZFC); EM: Llanos del Peñón IV (AMNH); Popocatépetl VI (USNM); Sierra Nevada (FES-Z: Luna-Reyes \& Llorente-Bousquets 2004); IXS: Ixtapan de la Sal III (CNIN); MALI: Chalma (Guzmán 1976); Camino viejo a Ocuilan VII, Colonia Juárez VI, El Molino IX, La Angostura VI, Puente Caporal XI-XII (CNIN: Hernández-Mejía 2005); OA: Ocuilan-Cuernavaca, km 14 I,III (MZFC); TEJ: Cañada La Canoa, 2.5 km del Reparo (Barrera \& Díaz-Batres 1977); ZAC: Zacualpan IX (MZFC); TOL: El Cerrillo Piedras Blancas VI.

Phyciodes graphica vesta (W.H. Edwards 1869). ALQ: Almoloya de Alquisiras IX (MZFC); ATZ: Atizapán (MCLB); AZ: Atizapán de Zaragoza VI-VIII (MZFC); JIL: Jilotepec X (CNIN); MALI: Chalma (Guzmán 1976); TEO: Cerro Gordo, Teotihuacán IV-V (CNIN).

Phyciodes mylitta thebais Godman \& Salvin 1878. EM: El Polvorín I (MZFC); Lagunas de Zempoala IV (CNIN), III (AMNH); VG: Villa Guerrero VIII (CNIN).

*Phyciodes pallescens (R. Felder 1869). ALQ: Almoloya de Alquisiras III (CNIN); CHIA: Tenancingo XI (CNIN); EM: Sierra Nevada (FES-Z: Luna-Reyes \& Llorente-Bousquets 2004); MALI: Chalma (Guzmán 1976); Colonia Juárez IIIIV, El Molino IV, Puente Caporal XII, Tepehuajes V (CNIN: Hernández-Mejía 2005); OA: Ocuilan-Cuernavaca, km 14 I,III (MZFC); VG: Villa Guerrero X (CNIN); ZAC: Puerto Obscuro II,XI (MZFC); Tres Cruces de Mamatla II (MZFC).

Phyciodes phaon phaon (W.H. Edwards 1864). MALI: Chalma (Guzmán 1976).

Phyciodes pulchella pulchella (Boisduval 1852). EM: Toluca, W de Méx. 15, km 18 VIII (HOLLA).

Phyciodes tharos tharos (Drury 1773). IXS: Ixtapan de la Sal VI (MZFC); MALI: San Andrés Nicolás Bravo VI (CNIN: Hernández-Mejía 2005).

SUBFAMILIA HELICONIINAE

Euptoieta claudia daunius (Herbst 1798). AMEC: Amecameca X (MZFC), (Godman \& Salvin 1878-1901); ATZ: Ciudad A. López Mateos VIII (CNIN); AZ: Atizapán de Zaragoza VI-VII (MZFC); CUA: Cuautitlán III (CNIN); EM: Sierra Nevada (FES-Z: Luna-Reyes \& Llorente-Bousquets 2004); MALI: Colonia Juárez III (CNIN: Hernández-Mejía 2005); OA: Ocuilan-Cuernavaca, km 14 VI (MZFC).

Euptoieta hegesia hoffmanni Stichel 1938. EM: Sierra Nevada (FES-Z: Luna-Reyes \& Llorente-Bousquets 2004); MALI: Chalma (Guzmán 1976); Camino viejo 
a Ocuilan VII, El Molino IX (CNIN: Hernández-Mejía 2005); OA: OcuilanCuernavaca, km 14 I (MZFC); TEM: Parque Universitario "Las Orquídeas" III,XI (Aguilera-Ríos \& Hernández-Mejía, en prep.); VB: Colorines (De la Maza 1987).

Agraulis vanillae incarnata (Riley 1926). EM: Sierra Nevada (FES-Z: Luna-Reyes \& Llorente-Bousquets 2004); MALI: Colonia Juárez IV (CNIN: HernándezMejía 2005); TEJ: El Estanco, Sierra de Nanchititla (Barrera \& Díaz-Batres 1977); TEM: Parque Universitario "Las Orquídeas" V,XII (Aguilera-Ríos \& Hernández-Mejía, en prep.); VB: Colorines (De la Maza 1987).

Dione juno huascuma (Reakirt 1866). CHIA: Tenancingo VIII (MZFC); EM: Sierra Nevada (FES-Z: Luna-Reyes \& Llorente-Bousquets 2004); MALI: Cerro "El Zapote" VII, Colonia Juárez III, El Platanar XI (CNIN: Hernández-Mejía 2005); TEM: Parque Universitario "Las Orquídeas" V,XII (Aguilera-Ríos \& Hernández-Mejía, en prep.); VB: Colorines (De la Maza 1987); ZAC: Zacualpan IX (MZFC).

Dione moneta poeyii Buttler 1873. CHIA: Tenancingo VIII (MZFC); EM: Cascada Los Diamantes, San Rafael I-II (MZFC), (Barrera \& Romero 1986); Cuello Ventonillo, Popocatépetl I (MZFC); Sierra Nevada (FES-Z: Luna-Reyes \& Llorente-Bousquets 2004); MALI: Puente Caporal X (CNIN: Hernández-Mejía 2005); TEJ: Cañada Bosque Encantado (Barrera \& Díaz-Batres 1977); TEM: Parque Universitario “Las Orquídeas” II,XII (Aguilera-Ríos \& Hernández-Mejía, en prep.); VB: Valle de Bravo (De la Maza 1987).

Dryadula phaetusa (Linnaeus 1758). ALQ: Almoloya de Alquisiras V (MZFC).

Dryas iulia moderata (Riley 1926). AZ: Atizapán de Zaragoza VIII (CNIN); EM: San Nicolás I,IX (CNIN); Ixtapantongo (De la Maza 1987); MALI: El Platanar IX, La Angostura XI, San Andrés Nicolás Bravo IV,XI (CNIN: Hernández-Mejía 2005).

Heliconius charithonia vazquezae W.P. Comstock \& F.M. Brown 1950. EM: San Nicolás I,IX (CNIN); Sierra Nevada (FES-Z: Luna-Reyes \& LlorenteBousquets 2004); MALI: Chalma (Guzmán 1976); Colonia Juárez V, El Molino V, El Platanar II,V,VII, San Andrés Nicolás Bravo VI (CNIN: Hernández-Mejía 2005); TEJ: El Estanco, Sierra de Nanchititla (Barrera \& Díaz-Batres 1977); TEM: Parque Universitario "Las Orquídeas” I,III,VI,XI-XII (Aguilera-Ríos \& Hernández-Mejía, en prep.). 
